\title{
Estudio experimental sobre el comportamiento térmico de un nuevo tipo de techo-estanque para el enfriamiento pasivo en clima húmedo
}

\author{
Experimental study on the thermal behaviour of a new \\ type of roof pond for passive cooling in humid climate
}

\section{Eduardo Manuel González-Cruz Sabrina Isabella González-Garcia}

Eduardo Manuel González-Cruz Universidad del Zulia Maracaibo, Venezuela

\section{Sabrina Isabella González- \\ Universidad Internaci Andalucia Andalucia}

Recebido em 15/04/13 Aceito em 18/08/13

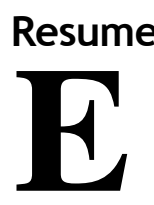

n este trabajo, una nueva tipología de techo-estanque metálico, basado en el enfriamiento evaporativo indirecto, que incluye masa térmica confinada, se evalúa experimentalmente. Cinco sistemas de enfriamiento pasivo se han estudiado con este dispositivo experimental. Se analizan sus temperaturas características con el propósito de determinar en cada caso, su potencial de enfriamiento, así como, la influencia de la masa térmica en su comportamiento térmico. Entre las técnicas de enfriamiento pasivo aplicadas se encuentran el enfriamiento evaporativo indirecto (EEI) y el enfriamiento radiativo nocturno (ER). El sistema donde se combina masa térmica con enfriamiento evaporativo indirecto en el dispositivo experimental presenta una elevada eficiencia de enfriamiento; su valor aumenta a medida que las condiciones son más extremas. Un potencial de enfriamiento aun mayor se consigue al agregar al sistema anterior enfriamiento radiativo.

Palavras-chave: Enfriamiento pasivo. Enfriamiento evaporativo indirecto.

Enfriamiento radiativo. Clima cálido húmedo. Masa térmica. Comportamiento térmico.

\section{Abstract}

In this paper, a new type of metal roof-pond based on indirect evaporative cooling and including confined thermal mass is experimentally evaluated. Five passive cooling systems have been studied with this experimental device. Their characteristic temperatures are analyzed in order to determine the cooling potential in each case and the influence of the thermal mass on thermal performance. The passive cooling techniques applied include indirect evaporative cooling (EEI) and nocturnal radiative cooling (ER). The system that combines thermal mass and indirect evaporative cooling in the experimental device presents high cooling efficiency; its value increases as the conditions become more extreme. A greater cooling potential is achieved by adding radiative cooling to the previous system.

Keywords: Experimental study. Passive cooling. Indirect evaporative cooling. Radiative cooling. Hot humid climate. Thermal mass. Thermal performance. 


\section{Introducción}

El calentamiento global es tal vez el mayor problema que enfrenta la sociedad a nivel mundial. Este fenómeno, de consecuencias impredecibles, es causado por el aumento de emisiones de gases de efecto invernadero. El sector edilicio es responsable en gran medida de esta problemática; buena parte de la energía que se consume está vinculada a este sector. En el caso norteamericano, como referencia, este sector es responsable del consumo del $49 \%$ del total de la energía utilizada en el país. Índices semejantes están presentes en gran parte de los países más desarrollados.

Venezuela no es ajena a esta problemática energética. El consumo de energía eléctrica venezolano per cápita es el mayor en latinoamérica, con un consumo anual de 4.145 KWh/per. Según estadísticas de Caveinel (CÁMARA..., 2007) el mayor porcentaje del consumo que se realiza en el país corresponde a los sectores industrial y residencial; solo los sectores residencial y comercial representan el $42.9 \%$ de la electricidad consumida. En el estado Zulia el elevado consumo eléctrico, estimado en $75 \%$ de la factura, se le atribuye fundamentalmente al uso masivo de equipos de aire-acondicionado destinados a la climatización mecánica de edificaciones de muy baja calidad térmica. Las altas temperaturas existentes a lo largo de todo el año, aunado a altos niveles de humedad relativa y la necesidad de conseguir "adecuados" niveles de confort térmico, conspiran contra el uso racional de las fuentes convencionales de energía.

El diseño arquitectónico adecuado a las condiciones climáticas del entorno y la aplicación de técnicas de enfriamiento pasivo pueden contribuir de manera significativa a reducir el consumo de energía en las edificaciones. Experiencias locales han demostrado la capacidad de reducción de la temperatura interna con relación a la del ambiente exterior de ciertos sistemas de enfriamiento pasivo (GONZÁLEZ, 1989, 1997a, 1997b; RINCÓN; ALMAO; GONZÁLEZ, 2001; HINZ, 2006). Los trabajos experimentales de Eduardo González en este campo han conducido hasta la construcción de una vivienda bioclimática prototipo (VBP-1) en la ciudad de Maracaibo, donde se aplica un sistema de enfriamiento evaporativo indirecto (GONZÁLEZ et al., 2000; GONZÁLEZ et al., 2006; GIVONI; GONZÁLEZ, 2009; GIVONI, 2011). La evaluación de esta vivienda, desde el punto de vista del confort térmico, ha permitido concluir que la aplicación del sistema de enfriamiento pasivo permite alcanzar condiciones de confort hasta en un $81 \%$ (BRAVO; GONZÁLEZ, 2013) considerando éstas como la suma de las respuestas de percepción térmica "confortable" y "ligeramente con frío". Sólo el $19 \%$ de las respuestas han sido de ligeramente con calor, incluidos dos estudios de campo en los periodos de marzo y julio. Fórmulas predictivas desarrolladas para la VBP-1 han permitido evaluar la aplicabilidad del SPEEI, utilizado en la vivienda, bajo diferentes condiciones climáticas (KRÜGER et al., 2010).

En este trabajo se estudia la aplicación del enfriamiento evaporativo y el enfriamiento radiativo, basados en el aprovechamiento del techo como elemento constructivo en "contacto" con dos de los pozos térmicos disponibles: el aire ambiente y el cielo ó bóveda celeste.

El enfriamiento evaporativo indirecto se ha investigado en numerosas ocasiones en diferentes partes del mundo. En Israel, B. Givoni (1981) realiza un estudio experimental sobre enfriamiento evaporativo y radiativo de techos entre los cuales un techo-estanque sombreado. Varios estudios experimentales siguieron a esas primeras experiencias que son reportadas por Givoni (1994), donde propone un modelo matemático de un sistema de techo-estanque con agua, ventilado, sobre una losa de concreto.

También en Israel, Tang, Etzion y Erell (2003) y Tang y Etzion (2004, 2005) realizan investigaciones sobre enfriamiento evaporativo en techos-estanque donde se utiliza un estanque con sacos de yute pintados en color blanco, que flotan en la superficie del agua (RPWGB). Esta técnica de enfriamiento y control de la ganancia de calor resultó ser más eficiente que algunos sistemas considerados como los de mayor potencial de enfriamiento (Techo cubierto con bolsas de yute mojado y techo-estanque con aislamiento móvil) esto debido a la estratificación térmica que se forma en el interior del estanque.

Por otro lado, Kharrufa y Adil (2008) estudiaron en Irak la aplicación del techo-estanque sobre una losa de concreto para la refrigeración de edificios en climas cálidos y áridos. En los resultados se observa que existió una mejora en las temperaturas con relación a la temperatura exterior. Sin embargo, se aprecia que la losa de concreto no permite la liberación rápida del calor ganado desde el espacio interior.

En la Universidad de Colima, México, en clima cálido sub-húmedo, Sánchez (1993), estudió el comportamiento térmico de un sistema de techoestanque utilizando un módulo experimental de $1.60 \times 1.60 \times 0.95 \mathrm{~m}$ construido con bloques de adobe y techo en concreto de $8 \mathrm{~cm}$ de espesor. Los

150 Gonzalez-Cruz, E. M.; Conzalez-Garcia, S. I. 
resultados muestran la capacidad del sistema para reducir la temperatura interna respecto a la temperatura exterior.

Por otro lado, en relación al enfriamiento radiativo (ER) los lugares con mayor potencial para su utilización son aquellos donde existen cielos claros, baja humedad especifica y poco viento. Los lugares húmedos, nublados, con altas temperaturas del aire y fuertes vientos no presentan potenciales de enfriamiento alto. Sin embargo, su aplicabilidad ha sido evaluada también en Maracaibo en diversos trabajos experimentales (GONZÁLEZ, 1997a). En uno de los primeros estudios realizados por este autor (GONZÁLEZ, 1989), un sistema de techo-estanque, totalmente hermético y lleno de agua, expuesto al cielo durante la noche y protegido durante el día, resultó con la mayor eficiencia de enfriamiento en un amplio estudio experimental de diversas técnicas de enfriamiento pasivo realizado con módulos de escala reducida.

El logro de condiciones de confort en el interior de las edificaciones tiene entre sus requisitos el mantenimiento de niveles de temperatura dentro de un rango relativamente pequeño. La oscilación interna o amplitud de temperatura (DT) dentro de una edificación depende en gran medida de la cantidad de "masa térmica efectiva" con que cuente el edificio. Las construcciones ligeras presentan oscilaciones internas altas, muchas veces superiores a la temperatura del aire exterior. En cambio, las construcciones masivas, realizadas con materiales pesados y espesores de muros importantes, tienden a tener oscilaciones internas pequeñas, menores a la amplitud de la temperatura exterior. Por otro lado, la utilización de masa térmica en los edificios puede reducir la carga pico de enfriamiento de los mismos (BALARAS, 1996). Este autor señala también la importancia de la ubicación, la cantidad y el tipo de material utilizado como masa térmica, sobre el comportamiento térmico del edificio.

Entre los materiales comúnmente usados como masa térmica encontramos: los ladrillos de adobe, los bloques de arcilla, la tierra, las rocas, el hormigón y el agua; este último con el más alto valor de capacidad térmica volumétrica (prácticamente el doble de un concreto denso), menor relación de masa volumen y menor costo, además de las ventajas que supone su manipulación y la posibilidad de que usada como masa térmica pueda enfriarse evaporativamente. La conveniencia de las edificaciones "pesadas" o con gran "masa térmica" para lograr condiciones de confort en climas tropicales húmedos y semihúmedos, ha sido reivindicada o defendida por diversos autores en las últimas décadas (GONZÁLEZ, 1997a, SOEVARTO, 1999,
SZOKOLAY, 2000, DORNELLES; RORIZ, 2004).

El propósito de este trabajo es estudiar experimentalmente el comportamiento térmico de cuatro configuraciones de sistemas pasivos de enfriamiento, basado en un nuevo tipo de techoestanque para su aplicación en el enfriamiento pasivo de edificaciones en el clima cálido-húmedo de Maracaibo, Venezuela. Para ello se analiza: el comportamiento térmico de cada sistema a partir de sus temperaturas características y factores decrementales; el potencial de enfriamiento (PEM) de cada uno de los sistemas de climatización pasiva evaluados y la influencia de la masa térmica sobre el desempeño de los sistemas estudiados.

Se parte de la hipótesis de que, es posible lograr mayor eficiencia de enfriamiento y reducción de la amplitud de la temperatura interior en una nueva configuración de techo-estanque, basado en el enfriamiento evaporativo indirecto, que incluya masa térmica confinada, que con el techo-estanque simple sombreado, cuya masa térmica es solo la constituida por el agua en proceso de evaporación.

\section{Metodología}

\section{El clima de la ciudad de Maracaibo y las estrategias bioclimáticas}

La ciudad de Maracaibo se encuentra ubicada al noroeste de Venezuela. Está localizada a $10^{\circ} 40.5^{\text {' }}$ de latitud norte y $71^{\circ} 37.3^{\prime}$ de longitud oeste. Su clima es clasificado como caliente y húmedo. Caracterizado por temperaturas y humedades relativas que varían muy poco durante el año; sus valores medios van de $27.9{ }^{\circ} \mathrm{C}$ a $31.2{ }^{\circ} \mathrm{C}$ (Tmed=29.6) y $71 \%$ a $83 \% \quad$ (HRmed=78\%) respectivamente, con amplitudes de $5.2{ }^{\circ} \mathrm{C}$ a $7{ }^{\circ} \mathrm{C}$. Durante el año se observan dos períodos de viento bien definidos. El primero, con régimen de vientos alisios, en los meses de diciembre a abril, con velocidades medias de 3 a $5 \mathrm{~m} / \mathrm{s}$. El segundo, de mayo-noviembre, con vientos muy débiles, variables en dirección y velocidad con predominio de tiempo de calma, especialmente entre 8 a.m. y 3 p.m. La precipitación anual promedio varía entre 450 y $550 \mathrm{~mm}$ y la radiación global media diaria es de $4.2 \mathrm{kWh} / \mathrm{m}^{2}$. Esta data climática corresponde al año 1997 de la estación meteorológica del Instituto de Investigaciones de la Facultad de Arquitectura y Diseño de la Universidad del Zulia; estación que se encuentra dentro del área urbana de la ciudad de Maracaibo.

Como se puede apreciar en la Figura 1, las condiciones de temperatura y humedad registradas a lo largo de todo el año se encuentra más de un $90 \%$ fuera del rango de confort indicado sobre 
carta psicrométrica. Por esta razón, se hace necesario utilizar estrategias de diseño bioclimático e implementar diferentes técnicas de enfriamiento pasivo para restaurar la condición de confort tanto como sea posible. Existen diferentes estrategias de enfriamiento y/o adecuación climática que ayudan a mejorar las condiciones de confort dentro de las edificaciones. La ventilación natural acompañada de la protección solar son las principales estrategias a utilizar en el clima de la ciudad de Maracaibo, sin embargo, no resulta suficiente aun cuando se contara con ventilación natural. Mediante el uso de la ventilación natural o mecánica se logran porcentajes de confort entre $80 \%$ y $87 \%$ en los meses de diciembre a abril, pero durante los meses más calientes del año se observan los valores más bajos, en torno al 50 $55 \%$ de confort, demostrando así lo anteriormente expresado.

De acuerdo con diversos programas de análisis de datos climáticos como el "Climate Consultant" (LIGGETT; MILNE, 2011) o el "Weather Tool" (MARSH, 2005), la aplicación de la estrategia de enfriamiento evaporativo indirecto en este clima no es recomendable pues no se consiguen porcentajes de confort significativos. Solo en los meses de diciembre a marzo puede representar entre $5 \%$ y $12 \%$ de confort térmico. Se trata de una estrategia que, vista de forma aislada, no ofrece resultados satisfactorios de acuerdo con este análisis. De igual manera sucede con el uso de masa térmica. Estas observaciones están en conformidad con la mayoría de la literatura en relación con las recomendaciones de diseño para climas cálidos y húmedos.

Entonces, ¿Porqué razón continuar investigando sobre la aplicación de sistemas basados en el enfriamiento evaporativo indirecto y el uso de masa térmica? La respuesta es que, experimentalmente se han logrado valores significativos de potencial de enfriamiento y de reducción de la temperatura interior respecto a la exterior. Además, se ha comprobado, con la evaluación del SPEEI de la vivienda bioclimática prototipo (VBP-1) y de los estudios de confort térmico dentro de la misma, que la combinación de estrategias de control de la ganancia de calor, masa térmica y de enfriamiento evaporativo indirecto, ha resultado muy favorable para los usuarios de la vivienda. Esto motiva a seguir investigando sobre modos más eficientes de utilizar el enfriamiento pasivo.

Figura 1 - Datos horarios de temperatura y humedad de Maracaibo y su relación con la zona de confort y las estrategias bioclimáticas sobre carta psicrométrica

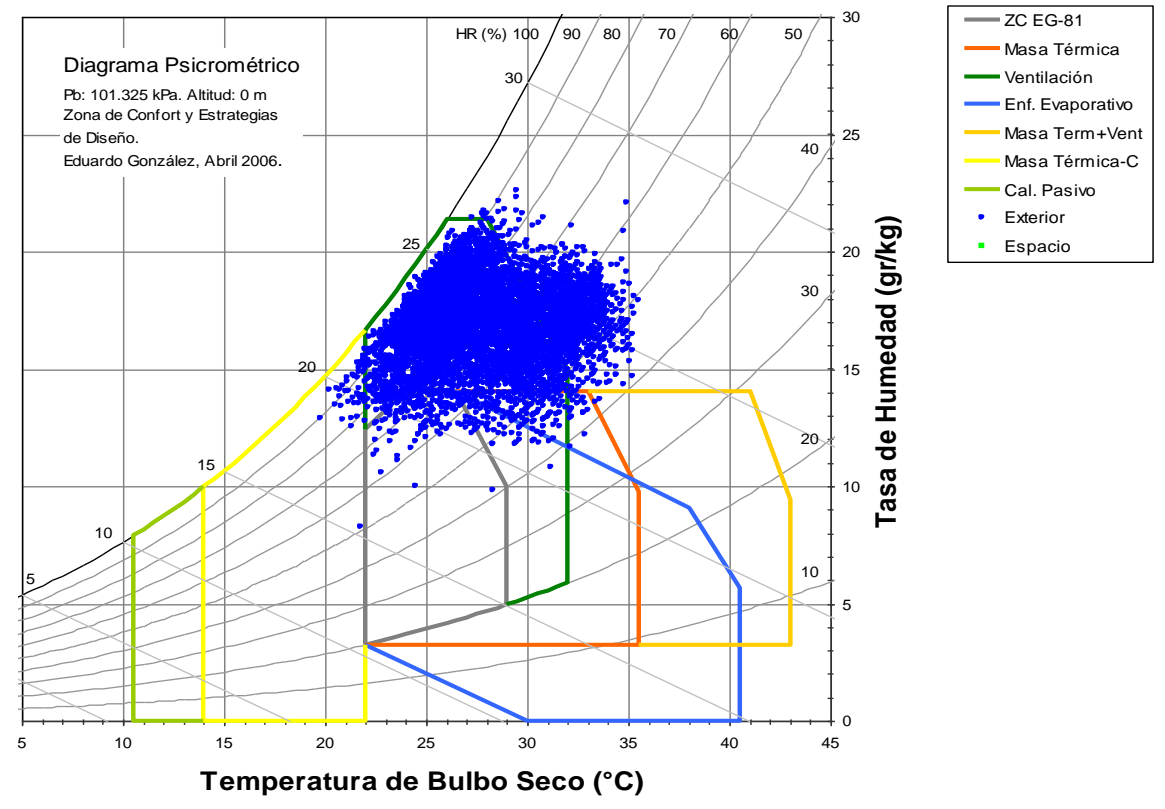

152 Gonzalez-Cruz, E. M.; Conzalez-Garcia, S. I. 


\section{Descripción del montaje experimental}

El montaje experimental consta de dos módulos de trabajo: un Módulo de Control (MC) y un Módulo Experimental (ME) (Figura 2 y 3). Ambos módulos son idénticos en forma, tamaño, dimensión, materiales y color exterior, salvo por el techo que los diferencia. De esta manera, la carga térmica a través de paredes y piso es igual en cada uno de ellos, con el fin de poder comparar el comportamiento del ME frente al MC y determinar el potencial de enfriamiento en cada uno de los experimentos a realizar. Las medidas utilizadas son de $0.80 \mathrm{~m}$ de largo por $0.80 \mathrm{~m}$ de ancho y 0.47 $\mathrm{m}$ de alto.

La fabricación de ambos módulos, se hizo con madera contra-enchapada de $0.015 \mathrm{~m}$ de espesor pintada en su exterior con pintura epóxica de color blanco. Fueron forrados internamente con láminas de poliestireno expandido de $0.045 \mathrm{~m}$ de espesor. Se colocaron dos listones de madera de $0.04 \mathrm{~m} \mathrm{x}$ $0.05 \mathrm{~m}$ en su base, con el fin de separarlos del suelo y evitar el contacto directo con el mismo.

\section{El Módulo de Control (MC)}

Con el propósito de reducir al máximo la ganancia de calor a través del techo, al módulo de control se colocó en la parte superior un aislamiento térmico de poliestireno de $0.15 \mathrm{~m}$ de espesor (Figura 4). Sus paredes se encuentran forradas por láminas de poliestireno de $0.045 \mathrm{~m}$ de espesor. En el interior se dejó un espacio libre de $0.30 \mathrm{~m}$ de alto donde se ubicó el sensor de medición de temperatura y humedad relativa.

\section{Figura 2 - Montaje experimental}

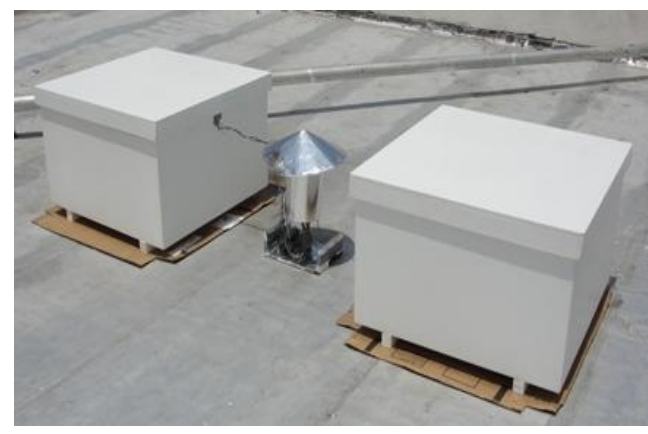

Figura 3 - Dimensiones de los módulos

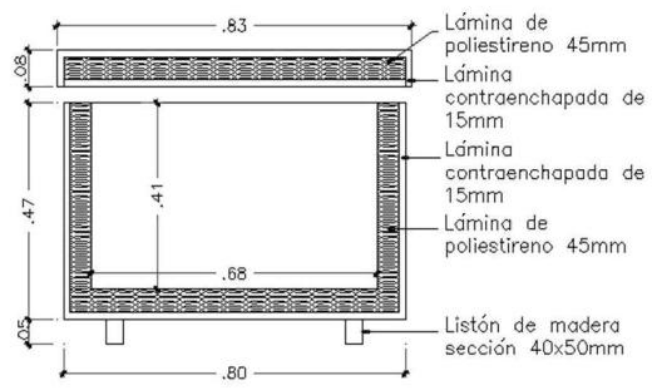

Figura 4 - Módulo de control
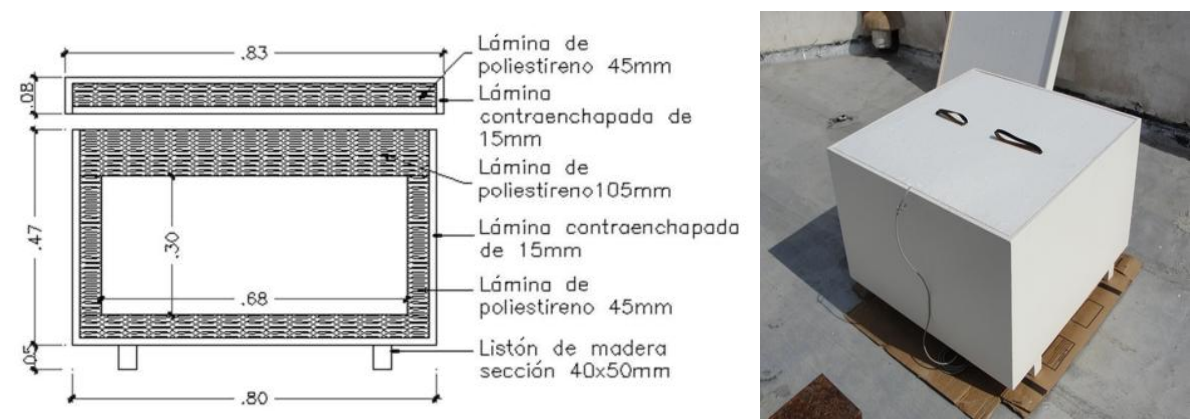


\section{Módulo experimental (ME)}

Al igual que el módulo de control, el módulo experimental (Figura 5) se encuentra aislado con $0.045 \mathrm{~m}$ de espesor de poliestireno en la parte inferior y paredes laterales. En su parte superior tiene un estanque elaborado en lámina galvanizada de $1.2 \mathrm{~mm}$ (calibre 18) y pintado en color blanco, dejando así un espacio interior libre de $0.30 \mathrm{~m}$ de alto, donde se colocaron sensores de medición de temperatura, humedad relativa y temperatura de globo. Se fabricaron dos tipos de cerramientos o cubiertas para el ME:

(a) una tapa de $0.83 \mathrm{~m} \times 0.83 \mathrm{~m}$ en madera contraenchapada de $0.015 \mathrm{~m}$, aislada con poliestireno de $0.045 \mathrm{~m}$ de espesor; $\mathrm{y}$

(b) un techo de protección solar de $1.00 \mathrm{~m} \times 1.00$ $\mathrm{m}$ en chapa de madera de $0.005 \mathrm{~m}$ aislada con una lámina de poliestireno de $0.015 \mathrm{~m}$ de espesor.

El cerramiento del módulo y los niveles de agua utilizados varían de acuerdo a cada experimento realizando. Esto se define al explicar cada caso.

\section{Descripción de equipos de medición}

Para el registro de temperaturas y humedad relativa se utilizaron seis equipos Hobo U12-001 Data Logger, comercializados por Onset (Figura 6). Los equipos presentan las siguientes características:

(a) 12 bit de resolución, provee una alta precisión; (b) rango de medición de $-20{ }^{\circ} \mathrm{C}$ a $70{ }^{\circ} \mathrm{C}$;

(c) precisión $\pm 0.35{ }^{\circ} \mathrm{C}$ de $0{ }^{\circ} \mathrm{C}$ a $50{ }^{\circ} \mathrm{C}$;

(d) resolución de $0.03{ }^{\circ} \mathrm{C}$ a $25^{\circ} \mathrm{C}$;

(e) tiempo de muestreo: 1 segundo hasta 18 horas, seleccionable por el usuario;

(f) tiempo de respuesta en movimiento de aire de $1 \mathrm{~m} / \mathrm{s}$ : 6 minutos, típico en $90 \%$;

(g) precisión de la hora: \pm 1 minuto por mes a 25 ${ }^{\circ} \mathrm{C}$;

(h) memoria de $64 \mathrm{~K}$ bytes; $\mathrm{y}$

(i) peso de 46 g. y unas dimensiones de $58 \times 74 \times 22 \mathrm{~mm}$

En cada uno de ellos se utilizó el canal externo para ubicar un sensor de medición TMC20-HD que registró la temperatura en interiores, exteriores y dentro del agua (Figura 7). El rango de medición de este sensor, es de $-40{ }^{\circ} \mathrm{C}$ a $50{ }^{\circ} \mathrm{C}$ en agua o en tierra y de $-40{ }^{\circ} \mathrm{C}$ a $100{ }^{\circ} \mathrm{C}$ en aire; el tiempo de respuesta en el agua es de un minuto y entre dos y tres minutos en el aire. Los equipos usan una interfase USB para descargar la información. Se realizó la calibración de los sensores para lo cual se colocaron bajo las mismas condiciones térmicas, en un contenedor de poliestireno, cerrado durante un día completo. La distribución general de los equipos y sensores de medición de los módulos y de las condiciones externas puede verse en la Figura 8.

Figura 5 - Módulo experimental
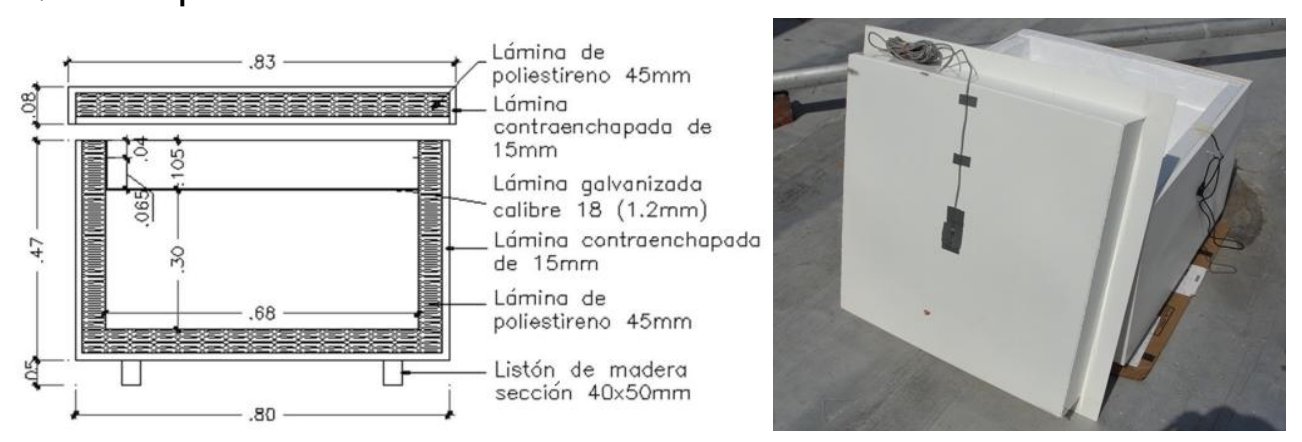

Figura 6 - Equipo registrador Hobo U12-001

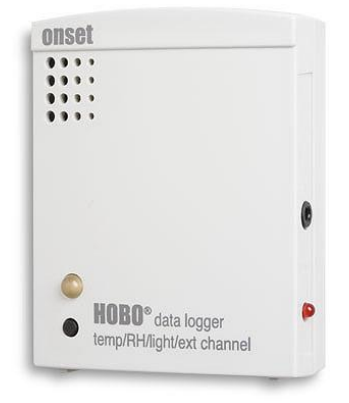

154 Gonzalez-Cruz, E. M.; Conzalez-Garcia, S. I. 
Figura 7 - Sensor TMC20-HD USB

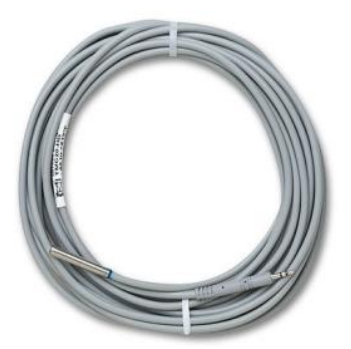

Figura 8 - Distribución de registradores y sensores en el MC y en el ME

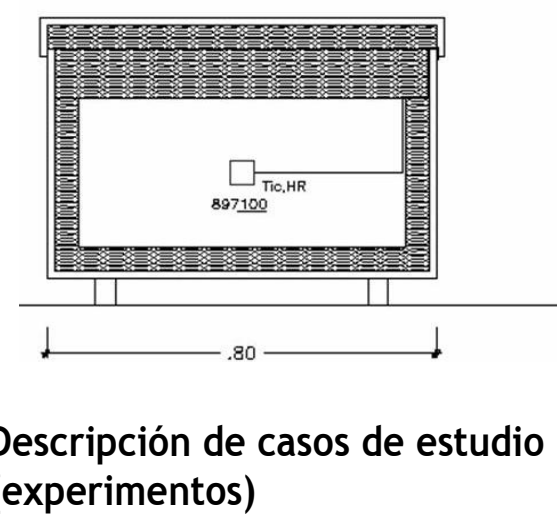

A continuación se describe la configuración del módulo experimental en cada uno de los casos estudiados de acuerdo con el objetivo de la evaluación y el modo de funcionamiento durante el periodo de monitoreo. Nos referiremos a ellos como los experimentos $3,4,5,6$ y 7 .

\section{Experimento 3: enfriamiento evaporativo indirecto y protección solar}

El experimento 3 tiene como objetivo evaluar el comportamiento del sistema de enfriamiento evaporativo indirecto. Para esto se coloca una altura de agua de $0.065 \mathrm{~m}$ en el techo-estanque metálico (Figura 9). El mismo, es protegido del sol por una lámina contraenchapada de madera de $0.005 \mathrm{~m}$, con una dimensión de $1.00 \mathrm{~m} \times 1.00 \mathrm{~m}$ pintada en color blanco y aislada en su cara interior con una lámina de poliestireno e $=0.015 \mathrm{~m}$. El protector solar se separa del módulo $0.06 \mathrm{~m}$, permitiendo de esta manera la ventilación del estanque de forma continua y su protección de la radiación solar debido al volado de $0.10 \mathrm{~m}$ existente en todo su alrededor (Figura 9). El monitoreo del sistema se realizó a lo largo de seis días. Se iniciaron las mediciones el día 7 de Septiembre de 2009, culminando el día 12 del mismo mes.

\section{Experimento 4: masa térmica y aislamiento térmico}

En el experimento 4 se quiere evaluar la influencia de la masa térmica bien aislada pero sin aplicación de ninguna técnica de enfriamiento pasivo. Para ello se cierra el techo-estanque con una lámina metálica a la altura de $0.065 \mathrm{~m}$, se sella con silicón y se llena de agua completamente, teniendo cuidado de que no queden burbujas de aire en su interior. Queda así una masa de agua de $\mathrm{h}=0.065 \mathrm{~m}$ totalmente confinada en el dispositivo metálico (Figura 10). Por otro lado, permanece una cámara de aire de $\mathrm{h}=0.04 \mathrm{~m}$, entre el estanque y la cubierta con aislamiento térmico de $0.045 \mathrm{~m}$ de poliestireno como se muestra en la misma figura. Este experimento fue monitoreado durante cuatro días, desde el día 14 de Septiembre hasta el día 17 del mismo mes.

\section{Experimento 5: enfriamiento radiativo y masa térmica}

Este experimento tiene como objetivo evaluar el potencial de enfriamiento radiativo nocturno cuando se dispone de una adecuada masa térmica que aprovecha el efecto de enfriamiento . El módulo experimental permanece como en el experimento número 4 , solo se modifica su funcionamiento. El ME permanece abierto (sin techo) durante las horas nocturnas, de 6:00pm a 6:00am (Figura 11). El resto de las horas del día se encuentra cerrado para evitar la ganancia de calor por radiación y convección. Este experimento fue monitoreado durante cincos días, del 19 al 23 de Septiembre del 2009. 
Figura 9 - Corte del ME en Experimento \#3 - enfriamiento evaporativo indirecto y protección solar
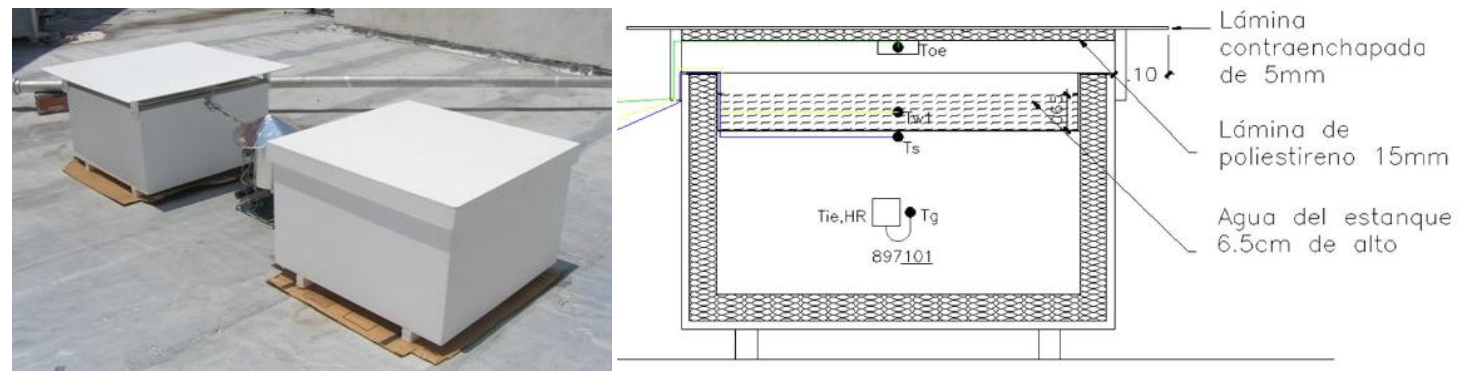

Figura 10 - Corte del ME en Experimento \#4 - masa térmica y aislamiento térmico 24h
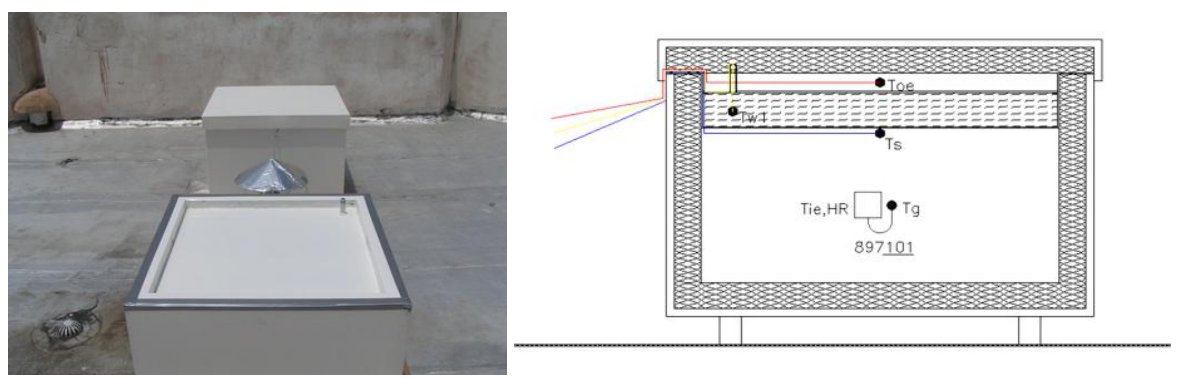

Figura 11 - Corte del ME en Experimento \#5 - enfriamiento radiativo y masa térmica - módulo cerrado de 6:00AM a 6:00pm (día) y abierto de 6:00pm a 6:00am (noche)

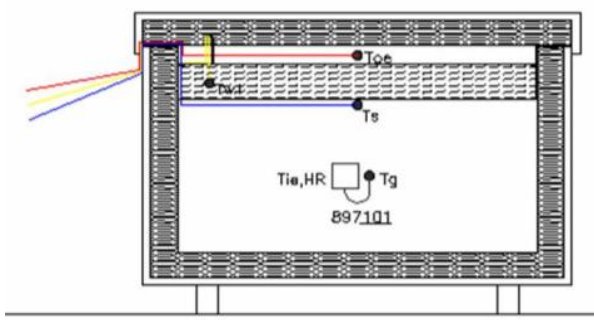

\section{Experimento 6: enfriamiento evaporativo indirecto, masa térmica y protección solar}

El experimento número seis, tiene como objetivo evaluar el techo-estanque con el sistema de enfriamiento evaporativo indirecto, combinado con la masa térmica (agua confinada en el dispositivo) y la protección solar. Esto es lo que se considera un "nuevo tipo de techo-estanque". En este caso, se realizaron tres series de mediciones de siete días cada una. La primera serie de mediciones dio inicio el día 27 de Septiembre y se extendió hasta el 3 de Octubre. Las siguientes dos series de mediciones se realizaron del 13 al 19 de Octubre y del 20 al 26 del mismo mes. De esta manera se monitoreó el sistema durante veintiún días, bajo condiciones climáticas diferentes.

El ME fue preparado con el estanque de agua lleno y cerrado para generar la masa térmica $(0.065 \mathrm{~m}$ de altura de agua -30 lts de agua). Se colocó agua sobre el estanque con una altura de $0.035 \mathrm{~m}$ para el funcionamiento del sistema evaporativo indirecto y

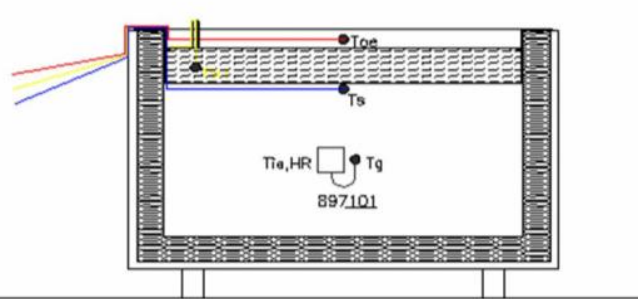

por último se colocó el techo de protección solar que permite la circulación de aire sobre el estanque limitando la penetración de los rayos solares sobre el techo-estanque (Figura 12).

\section{Experimento 7: enfriamiento radiativo nocturno, enfriamiento evaporativo indirecto, masa térmica y protección solar}

Este experimento tiene como objetivo evaluar el mismo sistema estudiado en el experimento 6 pero agregando el enfriamiento radiativo nocturno. Para ello, el ME es "destapado" durante las horas nocturnas (6:00pm a 6:00am) con el fin de aprovechar el enfriamiento por el intercambio radiante entre la masa de agua y la bóveda celeste. De 6:00am a 6:00pm se cubre para protegerlo de la radiación solar y evitar la ganancia de calor (Figura 13). El monitoreo de este experimento se realizó en un lapso de 7 días, desde el día 5 de Octubre hasta el día 11 del mismo mes. 
Figura 12 - Corte del ME en Experimento \#6 - enfriamiento evaporativo indirecto, masa térmica y protección solar

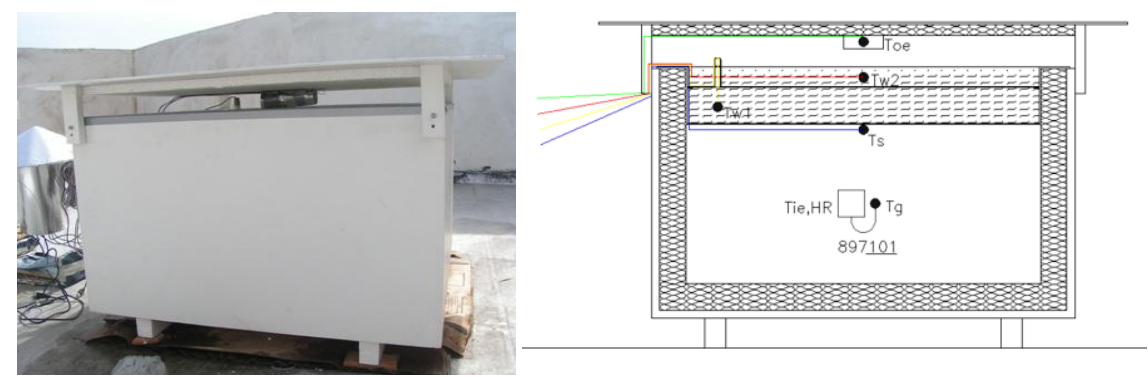

Figura 13 - Corte del ME en Experimento \#7 - enfriamiento radiativo nocturno, enfriamiento evaporativo indirecto, masa térmica y protección solar - módulo cerrado de 6:00am a 6:00pm (día) y abierto de 6:00pm a 6:00am (noche)
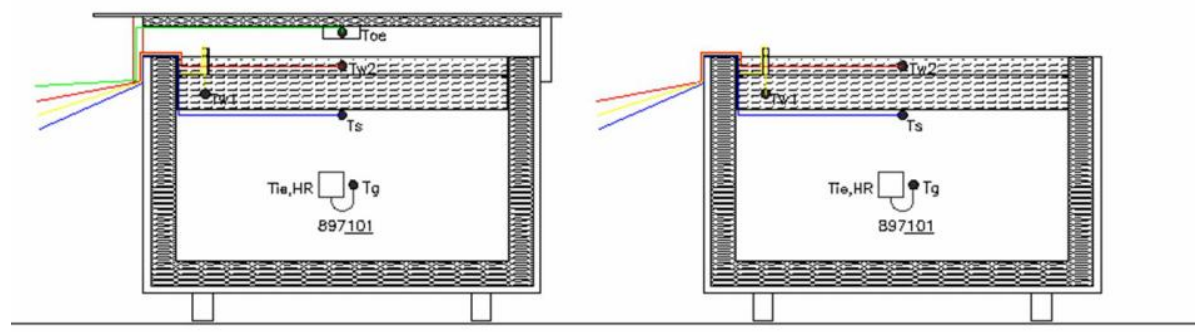

\section{Análisis de resultados}

\section{Análisis del comportamiento térmico: temperaturas características}

Las temperaturas características que aquí se evalúan corresponden a los valores:

(a) temperatura máxima (Tmax);

(b) temperatura mínima (Tmin);

(c) temperatura media (Tmed); y

(d) la amplitud de la temperatura (DT).

El análisis comparativo de estos valores en el módulo experimental con los valores correspondientes en el módulo de control y el ambiente exterior constituye la forma inicial de caracterizar el comportamiento térmico de dichos sistemas de enfriamiento.

Un sistema pasivo de enfriamiento será eficiente en la medida que logre reducir sus temperaturas máximas, medias y mínimas en relación con las temperaturas del módulo de control. Cuanto mayor sean estas diferencias mayor será la eficiencia de enfriamiento. En segundo lugar, es importante lograr la menor amplitud de temperatura posible en el interior del módulo experimental. Cuanto menor sea el factor decremental y mayor la diferencia entre las temperaturas medias internas de los dos módulos, entonces, mejor será el comportamiento térmico del sistema

En la Figura 14 se presentan los valores de temperatura máxima, media y mínima exterior de cada uno de los días monitoreados. Se han trazado sobre ellas las líneas de tendencia correspondiente a cada grupo de datos, para enfatizar el hecho de que, las condiciones climáticas durante los dos meses de experimentos fueron gradualmente haciéndose menos calientes y más lluviosos. Se recuerda que los experimentos comenzaron el 30 de agosto (el mes más caliente del año) y culminaron el 26 de octubre (el mes más lluvioso del año).

\section{Experimento 3: módulo experimental con enfriamiento evaporativo indirecto $y$ protección solar}

La evaporación del agua, fenómeno que se produce en forma continua en el techo-estanque del ME, representa una pérdida de calor continua desde el estanque hacia el aire. El experimento, cuyo comportamiento térmico se analiza a continuación, tiene como objetivo central evaluar un sistema basado en el enfriamiento evaporativo indirecto, mediante la utilización de un techo-estanque sombreado y ventilado. 
Figura 14 - Temperaturas máximas (Tomax), medias (Tomed) y mínimas (Tomin) diarias del ambiente durante el periodo experimental (30/08 al 26/10/2009)

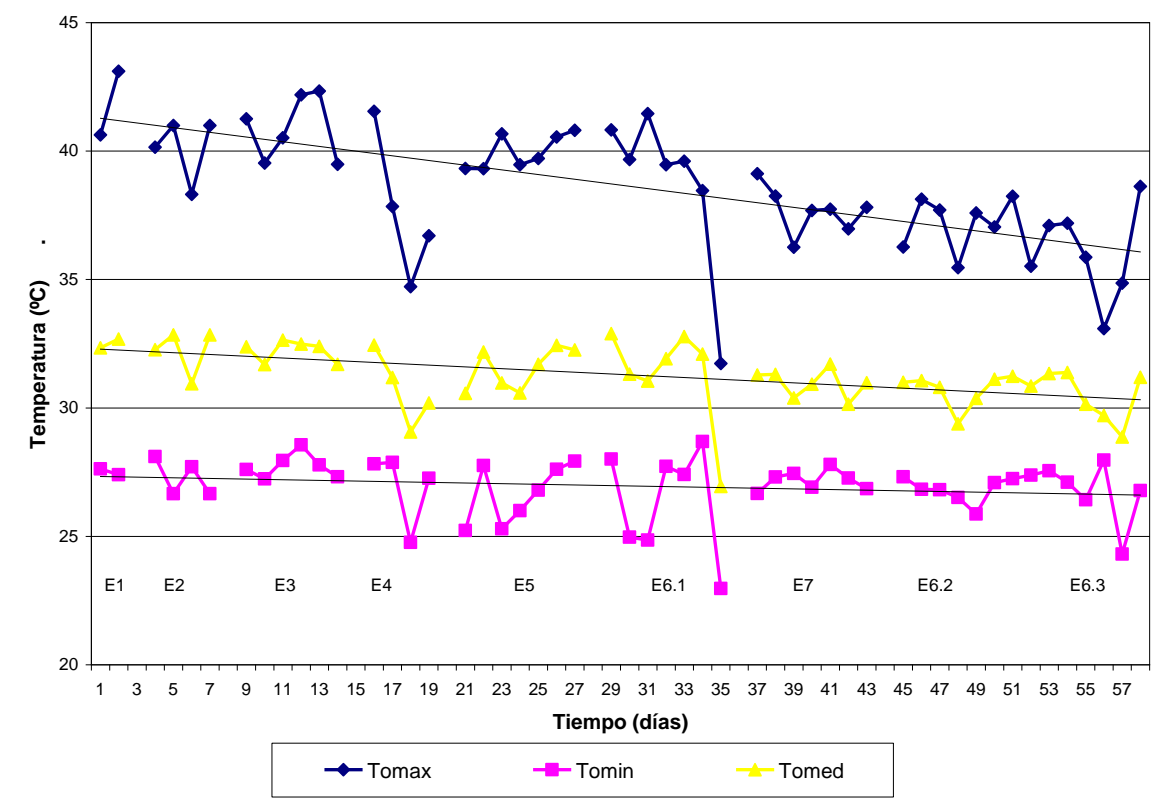

Tabla 1 - Temperaturas características experimento 3

\begin{tabular}{|c|c|c|c|c|c|c|c|c|c|c|c|c|c|}
\hline \multirow{2}{*}{ FECHA } & \multicolumn{5}{|c|}{ EXTERIOR (outdoor) } & \multicolumn{4}{|c|}{ MODULO CONTROL } & \multicolumn{4}{|c|}{ MODULO EXPERIMENTAL } \\
\hline & Tmax & Tmin & $\mathrm{Tm}$ & $\Delta T$ & $H R(m)$ & Tmax & Tmin & $\mathrm{Tm}$ & $\Delta \mathrm{T}$ & Tmax & Tmin & $\mathrm{Tm}$ & $\Delta T$ \\
\hline $08 / 09 / 2009$ & 39.5 & 27.2 & 31.7 & 12.3 & 62.4 & 39.1 & 26.5 & 31.2 & 12.6 & 30.1 & 24.5 & 26.9 & 5.6 \\
\hline $10 / 09 / 2009$ & 42.2 & 28.6 & 32.5 & 13.6 & 67.6 & 40.0 & 28.2 & 32.0 & 11.9 & 31.1 & 26.8 & 28.3 & 4.2 \\
\hline $11 / 09 / 2009$ & 42.3 & 27.8 & 32.4 & 14.6 & 64.3 & 39.0 & 27.1 & 31.9 & 11.8 & 30.0 & 26.0 & 27.7 & 4.0 \\
\hline $12 / 09 / 2009$ & 39.5 & 27.3 & 31.7 & 12.2 & 64.6 & 38.4 & 26.6 & 31.2 & 11.8 & 30.2 & 25.4 & 27.3 & 4.7 \\
\hline Media & 40.9 & 27.7 & 32.2 & 13.1 & 64.8 & 39.2 & 27.1 & 31.7 & 12.1 & 30.4 & 25.7 & 27.7 & 4.7 \\
\hline$\Delta \mathrm{T}$ & 2.8 & 1.3 & 0.9 & 2.4 & 5.2 & 1.6 & 1.6 & 0.9 & 0.8 & 1.2 & 2.3 & 1.4 & 1.6 \\
\hline
\end{tabular}

En la Figura 15 puede observarse la evolución de las temperaturas del ambiente, del MC y del ME, en los seis días de monitoreo de este experimento (7-12/09/2009). La temperatura interior en el ME (Tie) es todo el tiempo inferior a la temperatura exterior (To) y a la del MC (Tic). Se evidencia el notable descenso de la temperatura máxima en el módulo experimental en relación tanto con el MC como con la temperatura exterior; $8.8{ }^{\circ} \mathrm{C}$ en promedio inferior a la Tmax del MC (Tabla 1). Así mismo, se observa una caída de las temperaturas mínimas del ME respecto a la To y al MC; $1.4{ }^{\circ} \mathrm{C}$ en promedio inferior a la Tmin del MC. Como consecuencia de lo anterior la temperatura media del ME resulta en promedio $4.1{ }^{\circ} \mathrm{C}$ inferior a la temperatura media del $\mathrm{MC}$, lo que significa una alta eficiencia de enfriamiento considerando las condiciones climáticas calientes y húmedas de la región.

Otro aspecto muy importante a señalar es el relativo a la amplitud de la temperatura interna. En este caso, debido a la presencia de la masa térmica del agua, la oscilación de la temperatura interior es de tan solo $4.7{ }^{\circ} \mathrm{C}$ en promedio contra $12.1{ }^{\circ} \mathrm{C}$ en el MC. En este caso, el factor decremental promedio es 0.36 .

En síntesis, puede decirse que, este experimento demuestra el alto potencial de enfriamiento evaporativo indirecto de un sistema basado en un techo-estanque sombreado y ventilado, aun cuando las condiciones climáticas estén lejos de considerarse ideales para la aplicación de esta técnica de enfriamiento pasivo.

\section{Experimento 4: módulo experimental con masa térmica y aislamiento térmico}

Se ha considerado importante evaluar el efecto, que tiene el colocar una cierta cantidad de masa térmica en el techo, protegida con aislamiento térmico, sobre el comportamiento del módulo experimental. Con este propósito, el techoestanque fue dividido en dos partes: una inferior, llena de agua, completamente sellada con una

158 Gonzalez-Cruz, E. M.; Conzalez-Garcia, S. I. 
lámina metálica igual a la utilizada en la construcción del estanque, de 0.68 x 0.68 x 0.065 $\mathrm{m}$ con un volumen de 30.0 lts y otra superior de $0.68 \times 0.68 \times 0.04 \mathrm{~m}$. En este experimento, esta segunda zona del techo-estanque permanece vacía como una cámara de aire entre la masa térmica (agua) y el aislamiento térmico del techo.

En este experimento se constata el efecto de la masa térmica en tanto que reductor de la oscilación de la temperatura interna (Figura 16); la amplitud promedio del ME fue de $3.1{ }^{\circ} \mathrm{C}$, aproximadamente un tercio de la amplitud de $10.4{ }^{\circ} \mathrm{C}$ registrada en el MC (Tabla 2). Esta reducción de la amplitud interna es superior a la observada en el experimento 3, donde la misma masa de agua esta sometida a mayores flujos de calor. En este caso, el factor decremental promedio es 0.28 , menor al observado en el experimento 3 donde fue de 0.36 .

La temperatura interna media ha resultado prácticamente igual al $\mathrm{MC}$, tan solo $0.1{ }^{\circ} \mathrm{C}$ la diferencia entre ambos módulos. Aquí, como se observa, no hay enfriamiento del espacio interior respecto al MC, pero sí un control importante de la ganancia de calor por efecto del aislamiento térmico y en especial por el aislamiento por capacidad de la masa de agua con su alto calor especifico volumétrico.

Resulta interesante observar también en la Figura 16 la relación entre la temperatura interna del ME (Tie) y la temperatura del agua (Tw1) en el techoestanque sellado. El aumento y disminución de la Tie se ve limitado por la Tw1. Es el efecto de inercia térmica de la masa de agua que evita el calentamiento durante el día, pero también limita el enfriamiento durante la noche. De tal forma que, dadas las condiciones idénticas exteriores de los dos módulos, y sin presencia de alguna fuente de enfriamiento adicional, el efecto de la masa térmica es tan solo el de reducir la amplitud de temperatura respecto al $\mathrm{MC}$.

Figura 15 - Experimento 3: Evolución de temperaturas: exterior (To), bulbo húmedo exterior (Tbho), módulo de control (Tic), módulo experiemntal (Tie) y del agua en techo (Tw1) durante los días 7-12/09/2009

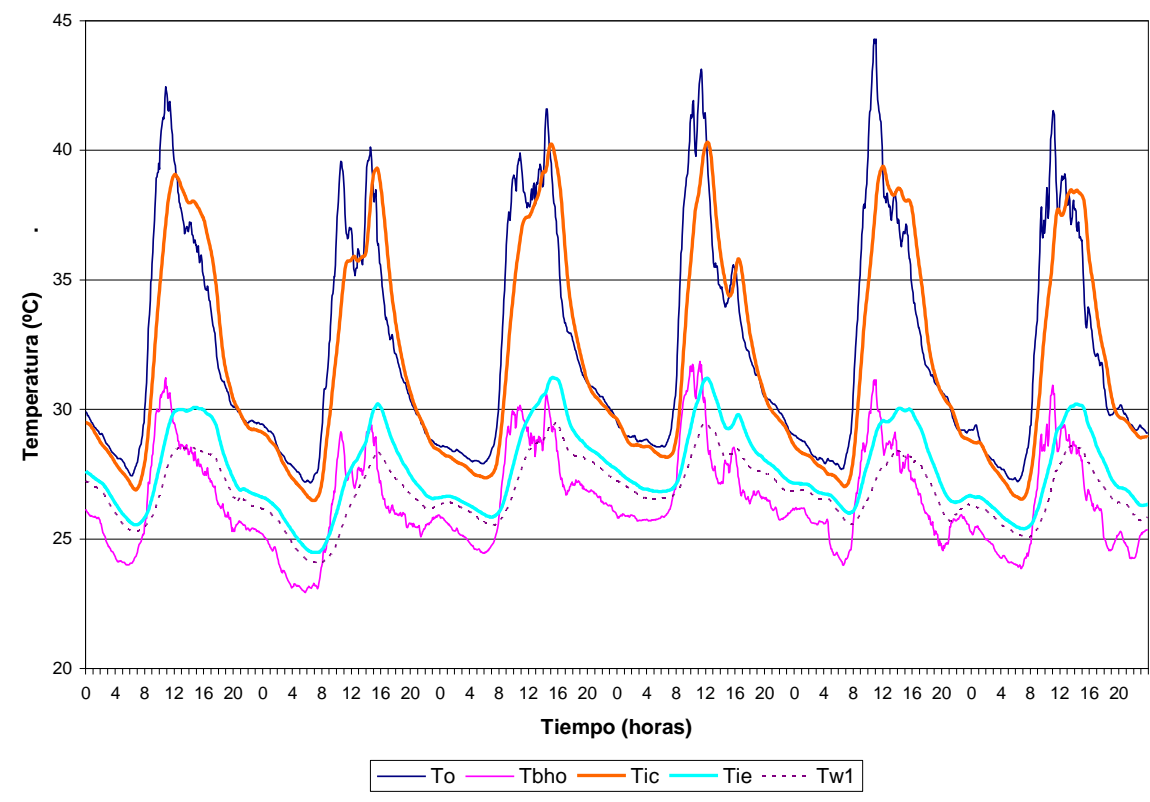

Tabla 2 - Temperaturas características experimento 4

\begin{tabular}{|c|c|c|c|c|c|c|c|c|c|c|c|c|c|}
\hline \multirow{2}{*}{ FECHA } & \multicolumn{5}{|c|}{ EXTERIOR (outdoor) } & \multicolumn{4}{|c|}{ MODULO CONTROL } & \multicolumn{4}{|c|}{ MODULO EXPERIMENTAL } \\
\hline & Tmax & Tmin & $\mathrm{Tm}$ & $\Delta \mathrm{T}$ & $\mathrm{HR}(\mathrm{m})$ & Tmax & Tmin & $\mathrm{Tm}$ & $\Delta \mathrm{T}$ & Tmax & Tmin & $\mathrm{Tm}$ & $\Delta \mathrm{T}$ \\
\hline $14 / 09 / 2009$ & 41.5 & 27.8 & 32.4 & 13.7 & 64.3 & 40.7 & 27.2 & 31.8 & 13.5 & 33.7 & 29.4 & 31.2 & 4.3 \\
\hline 15/09/2009 & 37.8 & 27.9 & 31.2 & 10.0 & 68.3 & 37.0 & 27.4 & 30.8 & 9.6 & 32.8 & 29.9 & 31.2 & 2.9 \\
\hline $17 / 09 / 2009$ & 36.7 & 27.3 & 30.2 & 9.4 & 70.4 & 35.5 & 26.7 & 29.8 & 8.8 & 31.2 & 28.4 & 29.7 & 2.7 \\
\hline Max & 41.5 & 27.9 & 32.4 & 13.7 & 70.4 & 40.7 & 27.4 & 31.8 & 13.5 & 33.7 & 29.9 & 31.2 & 4.3 \\
\hline Min & 34.7 & 24.8 & 29.1 & 9.4 & 64.3 & 34.4 & 24.7 & 29.0 & 8.8 & 30.9 & 28.4 & 29.7 & 2.4 \\
\hline
\end{tabular}


Figura 16 - Experimento 4 - Evolución de temperaturas: exterior (To), bulbo húmedo exterior (Tbho), módulo de control (Tic), módulo experiemntal (Tie) y del agua en techo (Tw1) durante los días 14-17/09/2009

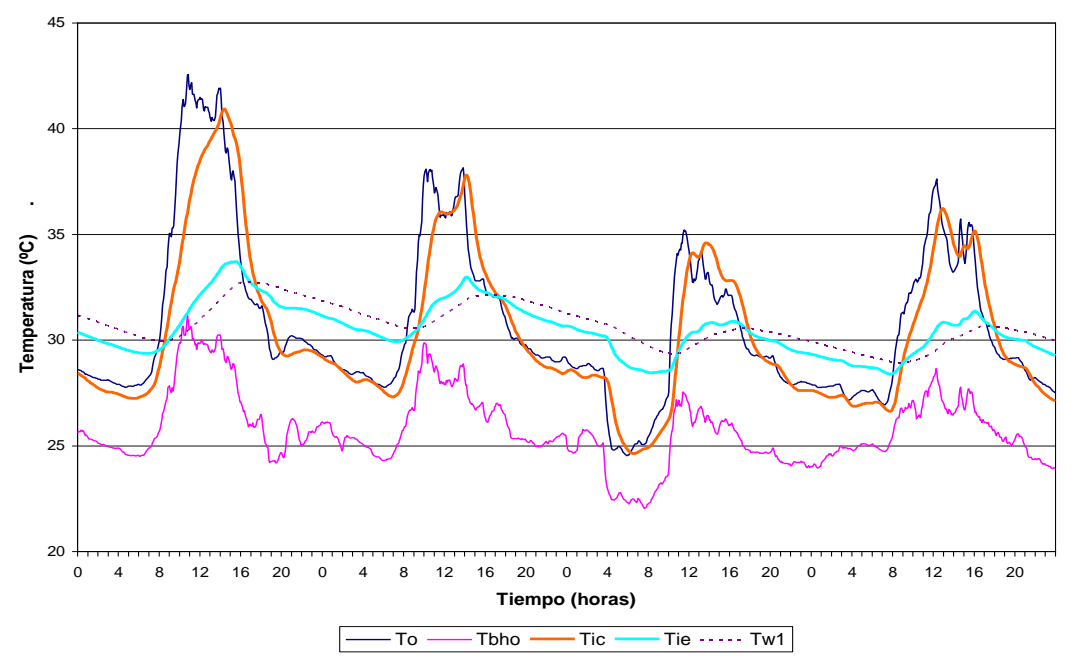

\section{Experimento 5: módulo experimental con masa térmica y enfriamiento radiativo}

En este caso, el ME que en el experimento anterior permanecía cerrado las $24 \mathrm{~h}$ del día, es "destapado" en la noche para exponer el techo-estanque al cielo nocturno y "tapado" con su aislamiento térmico durante las horas diurnas. De esta forma se estudia el comportamiento térmico del ME con masa térmica, constituida por 30 lts de agua contenidos en la parte inferior del techo-estanque, y enfriamiento radiativo.

La comparación de los resultados de este caso con el experimento 4 pone de manifiesto la importancia de contar con una masa térmica que aprovecha el enfriamiento radiativo nocturno para reducir su temperatura, limitando el aumento de la temperatura interior durante las horas diurnas. En este caso, se observa un efecto de enfriamiento neto importante, representado por una diferencia promedio entre las temperaturas medias internas de los módulos del orden de $2.5{ }^{\circ} \mathrm{C}$. En relación a la temperatura mínima, como se observa en la Figura 17, durante las horas nocturnas la temperatura en el ME (Tie) es prácticamente igual a la temperatura del agua (Tw1) dentro del techoestanque. Ambas temperaturas alcanzan valores mínimos iguales o ligeramente inferiores al MC (Tabla 3), mientras que, en el caso sin enfriamiento, el promedio fue $2.5{ }^{\circ} \mathrm{C}$ superior al MC (Tabla 2). Durante las horas diurnas, la masa enfriada en la noche cumple su rol al impedir que la temperatura interna aumente como en el caso del $\mathrm{MC}$, y por el contrario se mantiene ligeramente superior a la temperatura del agua, $8{ }^{\circ} \mathrm{C}$ en promedio inferior a la Tmax del MC. Un tercer aspecto es la reducción de la amplitud que, en el presente caso, es en promedio de $5.2{ }^{\circ} \mathrm{C}$ $(\mathrm{FD}=0.39)$, contra $13.3{ }^{\circ} \mathrm{C}$ en el caso sin masa térmica $(\mathrm{FD}=1.05)$. Cuando se compara la amplitud media interior del $\mathrm{ME}$ en los experimentos 4 y 5 , vemos como pasa de $3.1^{\circ} \mathrm{C}$ $(\mathrm{FD}=29 \%)$ a $5.1{ }^{\circ} \mathrm{C}(\mathrm{FD}=0.39 \%)$. Esto como consecuencia del mayor flujo de calor en el agua debido al proceso de enfriamiento nocturno.

\section{Experimento 6: módulo experimental con masa térmica y enfriamiento evaporativo indirecto y protección solar}

El sistema que se evalúa en el presente experimento constituye el objeto central de este trabajo. El objetivo es evaluar el comportamiento térmico de un sistema pasivo de enfriamiento evaporativo indirecto (SPEEI) que cuente con masa térmica en el techo, pero que esta masa térmica no represente una reducción de la eficiencia de enfriamiento. Cuando se aplica el enfriamiento evaporativo indirecto (estanque de agua sombreado) sobre losas de concreto en condiciones desfavorables como el clima de la ciudad de Maracaibo, la eficiencia de enfriamiento es muy baja (GONZÁLEZ, 2005). Por el contrario, lo ideal es que la masa térmica se comporte como un buen conductor y almacenador de alta difusividad y alta efusividad térmica (GONZÁLEZ, 1997a). Se considera que una forma práctica y económica de integrar los objetivos de tener "buena masa térmica" al mismo tiempo de también una "buena transferencia de calor" es utilizando agua confinada en un contenedor metálico como se propone en este estudio.

En este caso el módulo experimental cuenta con el techo-estanque que contiene 30 lts de agua

160 Gonzalez-Cruz, E. M.; Conzalez-Garcia, S. I. 
confinados en su parte inferior $(65 \mathrm{~mm}$ de altura de agua dentro del contenedor metálico) y 11.56 lts de agua en la parte superior del techo $(25 \mathrm{~mm}$ de altura de agua) ventilada y cubierta con una lámina de contraenchapado de $5 \mathrm{~mm}$ de espesor, revestida por su parte inferior con una lámina de poliestireno de $20 \mathrm{~mm}$ de espesor, del mismo modo que en el experimento 3 .

El experimento 6 fue monitoreado durante tres series de siete días cada una, desde el día 27/09/2009 al 26/10/2009. En la Figura 18 se presenta la evolución de temperaturas durante la tercera serie de mediciones. Las condiciones climáticas durante este experimento fueron muy variables, desde días asoleados de cielo claro hasta días muy nublados con intensos chubascos.
Del análisis de las curvas de temperatura en la Figura 18, así como también en el resto de los días monitoreados en este experimento, se observa que la temperatura del $\mathrm{ME}$ es inferior a la temperatura exterior y a la del MC, salvo en las horas de lluvia en que tienden todas a igualarse. Se evidencia un notable descenso de la temperatura máxima del ME respecto al MC y a la temperatura exterior; 8.2 ${ }^{\circ} \mathrm{C}$ en promedio inferior a la Tmax del MC (Tabla 4). Se observa también una caída de las temperaturas mínimas del ME respecto a la To y al MC; $0.5{ }^{\circ} \mathrm{C}$ en promedio inferior a la Tmin del MC. El comportamiento de la temperatura mínima en el ME se ve regido por las temperaturas del agua en las dos zonas del techo-estanque (Tw1 y Tw2); la primera más estable corresponde al agua confinada y cerca del espacio enfriado.

Tabla 3 - Temperaturas características experimento 5

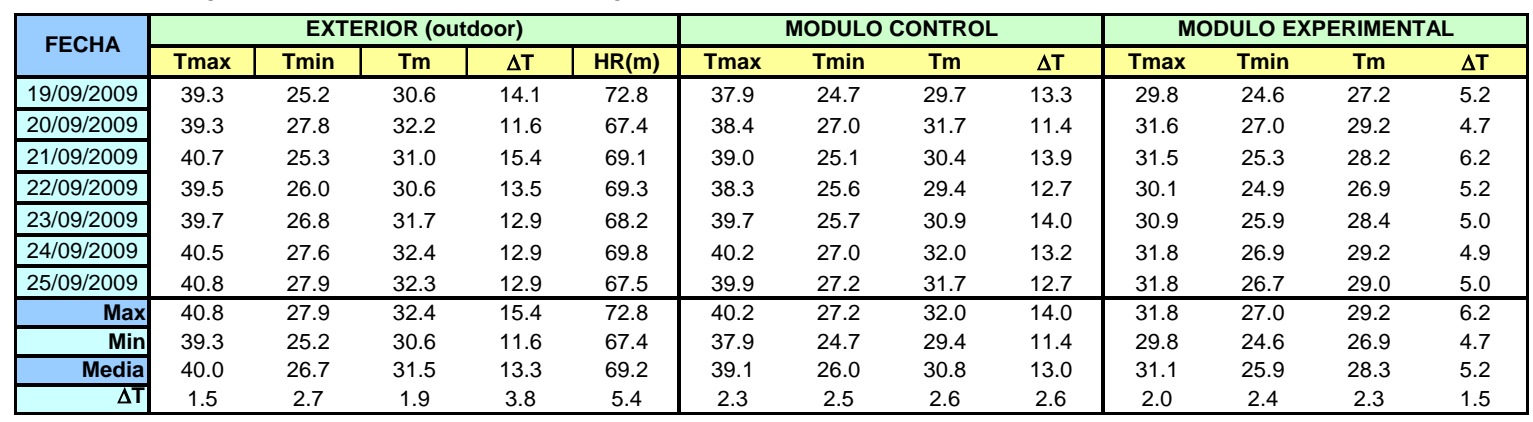

Figura 17 - Experimento 5. Evolución de temperaturas: exterior (To), bulbo húmedo exterior (Tbho), módulo de control (Tic), módulo experiemntal (Tie) y del agua en techo (Tw1) durante los días 19-25/09/2009

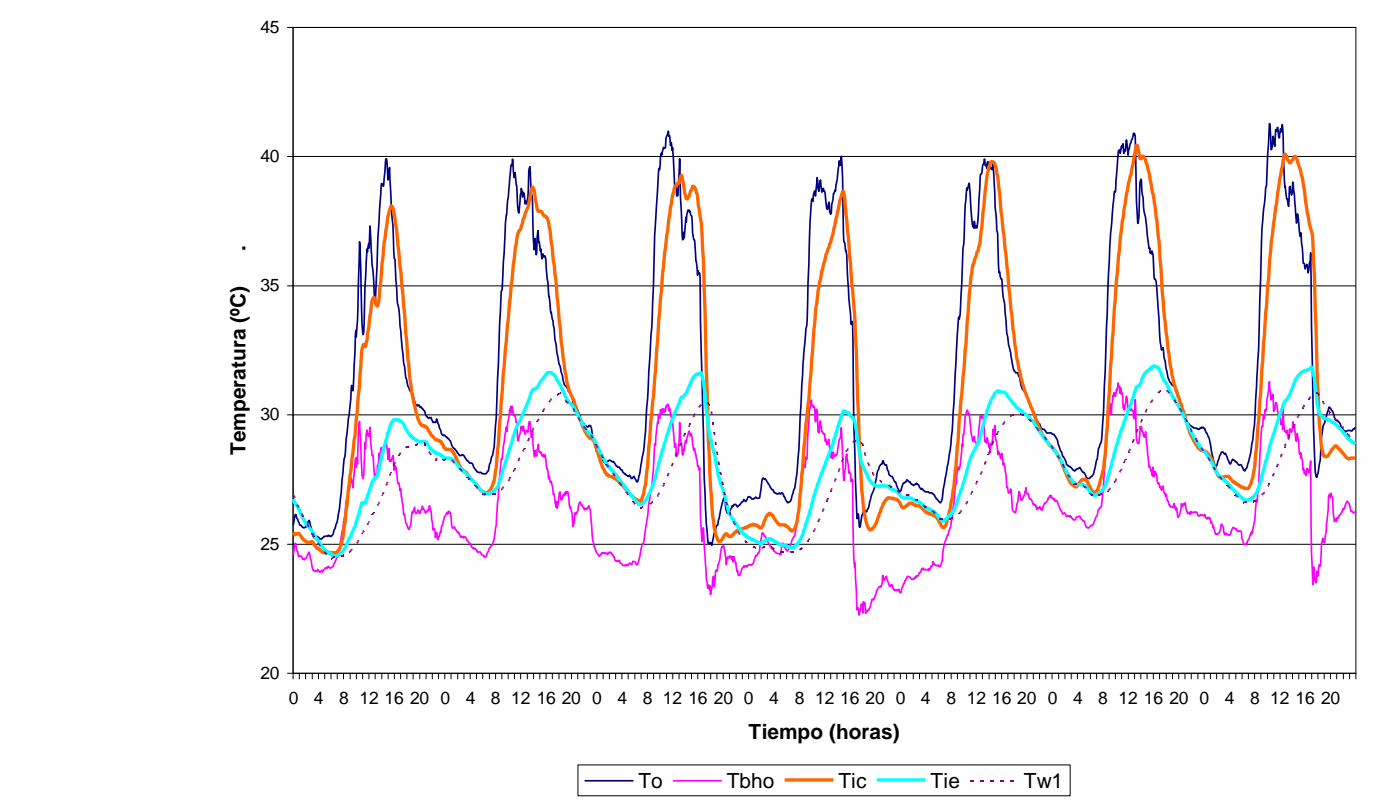

Tabla 4 - Temperaturas características experimento 6 (6.2)

Tabla 4 - Temperaturas caracteristicas experimento $6(6.2)$ 


\begin{tabular}{|c|c|c|c|c|c|c|c|c|c|c|c|c|c|}
\hline \multirow{2}{*}{ FECHA } & \multicolumn{5}{|c|}{ EXTERIOR (outdoor) } & \multicolumn{4}{|c|}{ MODULO CONTROL } & \multicolumn{4}{|c|}{ MODULO EXPERIMENTAL } \\
\hline & Tmax & Tmin & $\mathrm{Tm}$ & $\Delta \mathrm{T}$ & $\mathrm{HR}(\mathrm{m})$ & Tmax & Tmin & $\mathrm{Tm}$ & $\Delta \mathrm{T}$ & Tmax & Tmin & $\mathrm{Tm}$ & $\Delta \mathrm{T}$ \\
\hline 13/10/2009 & 36.3 & 27.3 & 31.0 & 9.0 & 64.9 & 37.4 & 26.8 & 31.1 & 10.7 & 29.0 & 25.3 & 26.9 & 3.7 \\
\hline 14/10/2009 & 38.1 & 26.8 & 31.1 & 11.3 & 65.0 & 39.1 & 26.1 & 31.0 & 12.9 & 29.4 & 25.1 & 27.0 & 4.4 \\
\hline $15 / 10 / 2009$ & 37.7 & 26.8 & 30.8 & 10.9 & 65.7 & 38.9 & 26.2 & 30.7 & 12.7 & 29.2 & 24.9 & 26.7 & 4.4 \\
\hline 16/10/2009 & 35.5 & 26.5 & 29.4 & 8.9 & 72.4 & 36.0 & 26.1 & 29.0 & 10.0 & 28.8 & 25.5 & 26.7 & 3.3 \\
\hline $17 / 10 / 2009$ & 37.6 & 25.9 & 30.4 & 11.7 & 72.5 & 38.4 & 25.2 & 30.2 & 13.3 & 29.7 & 25.4 & 27.2 & 4.3 \\
\hline $18 / 10 / 2009$ & 37.0 & 27.1 & 31.1 & 10.0 & 67.8 & 38.2 & 26.4 & 31.0 & 11.9 & 29.7 & 26.1 & 27.6 & 3.7 \\
\hline 19/10/2009 & 38.2 & 27.2 & 31.2 & 11.0 & 64.4 & 39.2 & 26.8 & 31.3 & 12.3 & 29.3 & 25.6 & 27.1 & 3.6 \\
\hline Max & 38.2 & 27.3 & 31.2 & 11.7 & 72.5 & 39.2 & 26.8 & 31.3 & 13.3 & 29.7 & 26.1 & 27.6 & 4.4 \\
\hline Min & 35.5 & 25.9 & 29.4 & 8.9 & 64.4 & 36.0 & 25.2 & 29.0 & 10.0 & 28.8 & 24.9 & 26.7 & 3.3 \\
\hline Media & 37.2 & 26.8 & 30.7 & 10.4 & 67.5 & 38.2 & 26.2 & 30.6 & 12.0 & 29.3 & 25.4 & 27.0 & 3.9 \\
\hline$\Delta \mathrm{T}$ & 2.8 & 1.4 & 1.9 & 2.8 & 8.1 & 3.2 & 1.7 & 2.3 & 3.3 & 0.9 & 1.2 & 0.9 & 1.1 \\
\hline
\end{tabular}

La temperatura media del $\mathrm{ME}$ ha resultado en promedio $3.3{ }^{\circ} \mathrm{C}$ inferior a la temperatura media del MC. Sin embargo, esa diferencia ha variado mucho durante el periodo de monitoreo. La diferencia máxima alcanzada ha sido $4.2{ }^{\circ} \mathrm{C}$ y como mínimo ha llegado a $1{ }^{\circ} \mathrm{C}$. La diferencia de Tmed entre el MC y el ME para los días sin precipitación ha sido en promedio de $4.0{ }^{\circ} \mathrm{C}$. Estas diferencias entre las temperaturas medias de los dos módulos expresan una importante eficiencia de enfriamiento que, aunado al notable descenso de la temperatura máxima en relación al $\mathrm{MC}$, permiten concluir que se trata de un sistema viable aún en estas condiciones calientes y húmedas tropicales.

\section{Experimento 7: módulo experimental con masa térmica, enfriamiento evaporativo indirecto, enfriamiento radiativo y protección solar}

Este último experimento tiene como objetivo evaluar el sistema pasivo de enfriamiento evaporativo indirecto con masa térmica, descrito en el experimento 6, pero apoyado adicionalmente en el aprovechamiento del enfriamiento radiativo nocturno. Con este propósito, se descubre el techoestanque del ME de 6:00pm a 6:00am y se deja cubierto entre 6:00AM a 6:00pm, con ventilación sobre el agua como en los experimentos 3 y 6 .

El análisis de la evolución de las temperaturas durante los días 5-11/10/2009 en la Figura 19 y de las temperaturas características que se presentan en la Tabla 5 permiten comprobar un comportamiento térmico del sistema semejante al observado en el experimento 3 y durante las tres series de monitoreo del experimento 6. Comportamiento que se caracteriza por: temperaturas máximas muy inferiores a las del MC y a las del ambiente exterior $\left(8.6{ }^{\circ} \mathrm{C}\right.$ en promedio inferior al $\left.\mathrm{MC}\right)$; temperaturas mínimas siempre inferiores al MC y al ambiente exterior $\left(1.2{ }^{\circ} \mathrm{C}\right.$ en promedio inferior al MC); temperaturas medias inferiores al MC y al ambiente exterior $\left(3.6{ }^{\circ} \mathrm{C}\right.$ en promedio inferior al MC) y amplitudes de temperatura interna en promedio de $4.1{ }^{\circ} \mathrm{C}(\mathrm{FD}=0.39)$ igualmente muy inferiores a las que se observan en el MC y en la temperatura exterior.

Es importante observar en estos resultados que la temperatura media del ME es prácticamente igual a la temperatura mínima del ambiente. Es notable la eficiencia de enfriamiento que se consigue con este sistema que, al contar con la posibilidad de enfriarse adicionalmente al exponer el agua del estanque al cielo nocturno, alcanza temperaturas medias internas inferiores en $0.2{ }^{\circ} \mathrm{C}$ en promedio al experimento 6 , sin enfriamiento radiativo.

\section{Análisis de factores decrementales}

Si bien se han reportado los valores promedio de los factores decrementales (FD) de cada uno de los experimentos, referidos al cociente entre la temperatura interna media del ME y la temperatura media del ambiente, conviene discutir brevemente el FD en relación con el MC (Tabla 6).

Comparado de este modo el FD, al tener como base la amplitud de la temperatura en el MC se constata el efecto de la masa térmica al reducir la amplitud de la temperatura interna del ME en relación al MC. El menor valor de FD (0.29) se observa en el caso donde la masa térmica se encuentra aislada y protegida las 24 horas del día (Experimento 4). Cuando la masa térmica es sometida a un proceso de enfriamiento, la oscilación de su temperatura es mayor. Así vemos, en los experimentos 3 y 5 , que los FD han aumentado a 0.39 y 0.40 respectivamente. Al aumentar la cantidad de masa térmica en el experimento 6 se observa una reducción de FD. En las tres series monitoreadas de este experimento el FD ha sido en promedio 0.34 , lo cual confirma una de las hipótesis del trabajo. Finalmente, el experimento siete presenta como promedio un FD de 0.36 , ligeramente superior al observado en el experimento 6. Debe entenderse esto como una consecuencia del mayor flujo de calor al adicionar el enfriamiento radiativo al experimento 6. 
Figura 18 - Experimento 6.2: Evolución de temperaturas: exterior (To), bulbo húmedo exterior (Tbho), módulo de control (Tic), módulo experiemntal (Tie) y del agua en techo (Tw1-Tw2) durante los días 13-19/10/2009

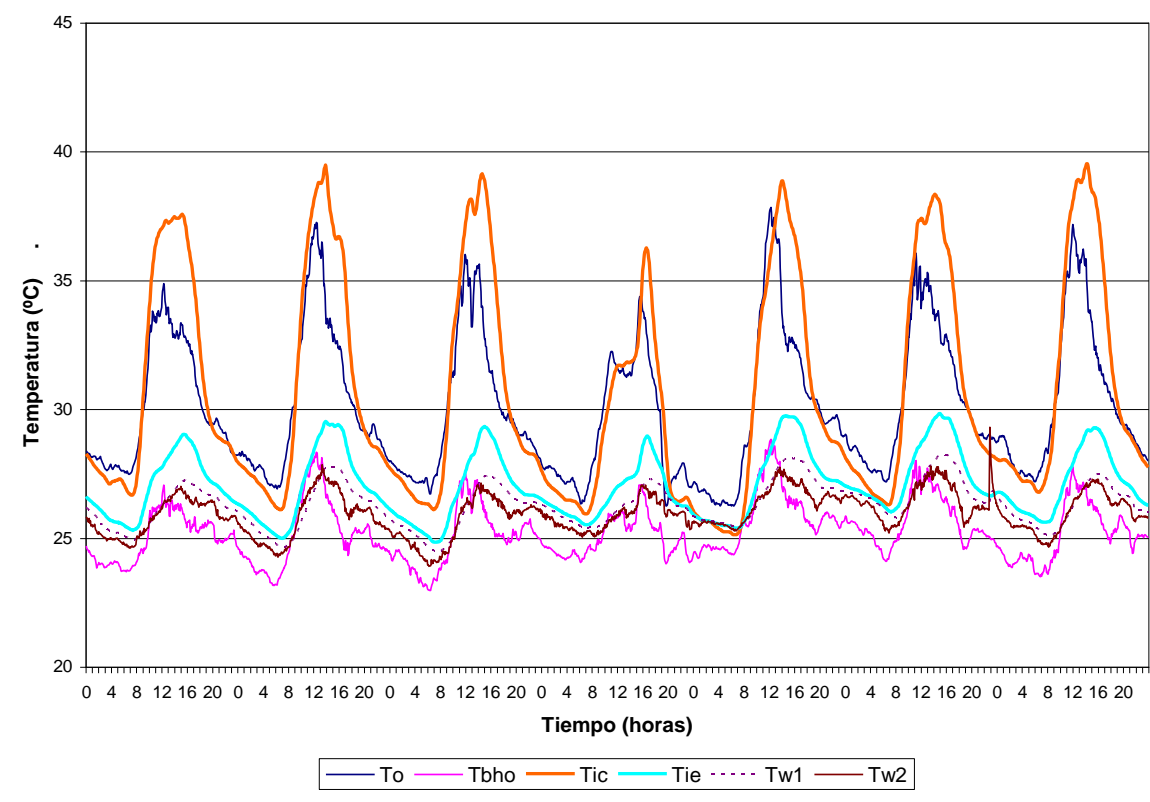

Tabla 5 - Temperaturas características experimento 7

\begin{tabular}{|c|c|c|c|c|c|c|c|c|c|c|c|c|c|}
\hline \multirow{2}{*}{ FECHA } & \multicolumn{5}{|c|}{ EXTERIOR (outdoor) } & \multicolumn{4}{|c|}{ MODULO CONTROL } & \multicolumn{4}{|c|}{ MODULO EXPERIMENTAL } \\
\hline & Tmax & Tmin & $\mathrm{Tm}$ & $\Delta \mathrm{T}$ & $\mathrm{HR}(\mathrm{m})$ & Tmax & Tmin & $\mathrm{Tm}$ & $\Delta \mathrm{T}$ & Tmax & Tmin & $\mathrm{Tm}$ & $\Delta \mathrm{T}$ \\
\hline $05 / 10 / 2009$ & 39.1 & 26.7 & 31.3 & 12.5 & 70.5 & 39.2 & 26.0 & 30.9 & 13.1 & 30.3 & 25.5 & 27.4 & 4.8 \\
\hline $06 / 10 / 2009$ & 38.2 & 27.3 & 31.3 & 10.9 & 67.1 & 38.4 & 26.6 & 31.4 & 11.8 & 29.4 & 25.2 & 27.2 & 4.2 \\
\hline $08 / 10 / 2009$ & 37.7 & 26.9 & 30.9 & 10.8 & 70.5 & 37.7 & 26.2 & 30.5 & 11.4 & 29.0 & 25.3 & 27.1 & 3.7 \\
\hline 09/10/2009 & 37.7 & 27.8 & 31.7 & 9.9 & 68.7 & 38.6 & 27.2 & 31.7 & 11.4 & 30.1 & 26.1 & 27.7 & 3.9 \\
\hline 10/10/2009 & 37.0 & 27.3 & 30.1 & 9.7 & 71.1 & 37.4 & 27.0 & 29.9 & 10.4 & 28.7 & 25.3 & 26.7 & 3.5 \\
\hline Min & 36.3 & 26.7 & 30.1 & 8.8 & 67.1 & 37.4 & 26.0 & 29.9 & 10.4 & 28.7 & 25.0 & 26.7 & 3.5 \\
\hline Media & 37.7 & 27.2 & 31.0 & 10.5 & 69.7 & 38.1 & 26.6 & 30.8 & 11.5 & 29.5 & 25.4 & 27.1 & 4.1 \\
\hline$\Delta \mathrm{T}$ & 2.9 & 1.1 & 1.6 & 3.6 & 4.0 & 1.8 & 1.1 & 1.8 & 2.8 & 1.6 & 1.1 & 1.0 & 1.3 \\
\hline
\end{tabular}

Figura 19 - Experimento 7: Evolución de temperaturas durante los días 5-11/10/2009

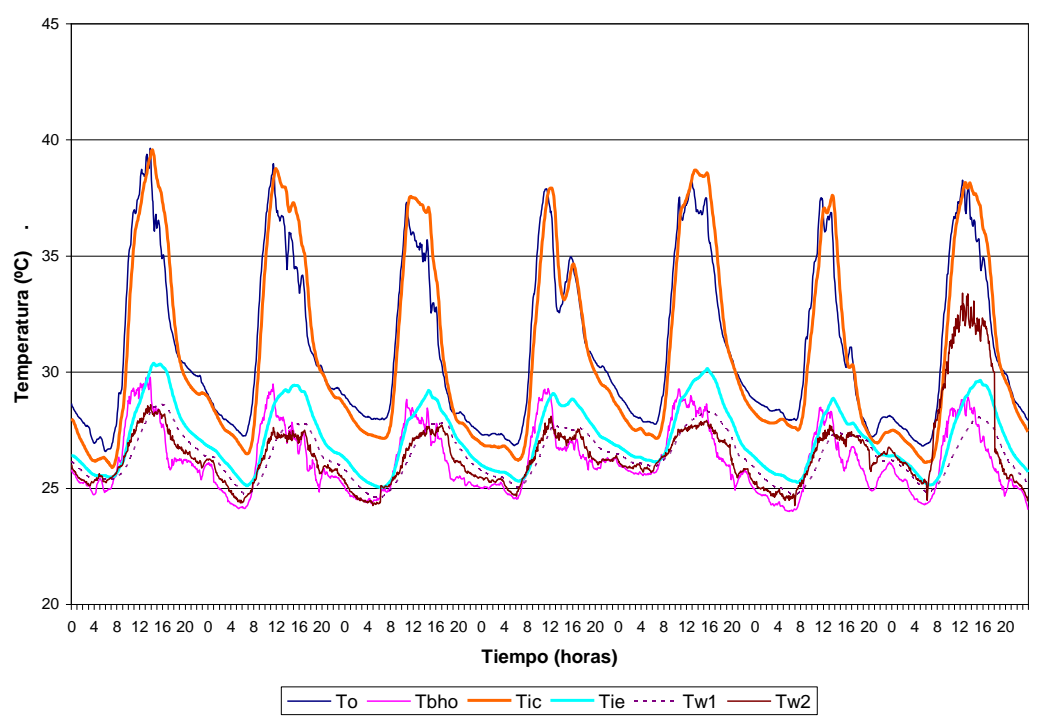


Tabla 6 - Factores decrementales en cada uno de los sistemas analizados

\begin{tabular}{c|l|c}
\hline Exp & \multicolumn{1}{|c}{ Configuración de Módulo experimental } & FD \\
\hline 3 & Masa térmica+enfriamiento evaporativo indirecto & 0.39 \\
4 & Masa térmica+aislamiento & 0.29 \\
5 & Masa térmica+enfriamiento radiativo & 0.40 \\
6 & Masa térmica+enfriamiento evaporativo indirecto & 0.34 \\
7 & Masa térmica+enfriamiento evaporativo indirecto + enfriamiento radiativo & 0.36 \\
\hline
\end{tabular}

\section{Correlación entre las temperaturas medias en el MC y el ME}

El análisis de correlación entre las temperaturas medias registradas en el MC y aquellas registradas en el ME nos permiten verificar la coherencia de los resultados y la eficiencia de enfriamiento, de manera comparativa y establecer líneas de tendencia para cada uno de los sistemas estudiados. En la Figura 20 se presentan las correlaciones entre las temperaturas medias del MC y las temperaturas medias del ME, correspondientes a cada experimento. En la misma figura se han trazado las líneas de tendencia o de regresión para cada conjunto de datos y los coeficientes de determinación (R2) de cada caso.

En el experimento 3 el análisis de regresión lineal nos indica que la diferencia entre Tic y Tie tiende a disminuir a medida que aumenta la temperatura media del MC, mientras que las líneas de tendencia en los experimentos 6 y 7 presentan una situación contraria; en estas, la diferencia entre el Tic y Tie aumenta a medida que Tic es mayor. A simple vista los datos del experimento 3, en relación a los del resto que se presentan, indican que es el caso donde hay mayor eficiencia de enfriamiento, pues corresponden a esta serie las mayores diferencias de temperatura entre el MC y el ME. Sin embargo, al analizar las líneas de tendencia podemos concluir que esto es cierto hasta ciertas condiciones climáticas donde Tic (MC) es igual o menor a $32{ }^{\circ} \mathrm{C}$ aproximadamente, de allí en adelante los sistemas evaluados en los experimentos 6 y 7 teóricamente alcanzarían diferencias de temperaturas mayores al caso 3 .

En el experimento 5, con enfriamiento radiativo, aumenta la diferencia de temperatura media entre los módulos a medida que aumenta la temperatura en el MC. Es decir, que la eficiencia de enfriamiento de este sistema se hace mayor con el aumento de la temperatura en el MC, o lo que es igual, con el aumento de la temperatura del ambiente exterior. En este caso el coeficiente de determinación es muy elevado, $\mathrm{R}^{2}=0.9791$ y la ecuación de la línea de tendencia es $\mathrm{Y}=0.8993 \mathrm{X}+$ 0.5724 .

En relación con el experimento 6 (6.1, 6.2 y 6.3), la tendencia es semejante al experimento 5 , es decir que, la eficiencia de enfriamiento aumenta al aumentar Tic. Sin embargo, la línea de tendencia tiene una pendiente mucho menor. En este caso, el coeficiente de determinación es medio-alto $\mathrm{R}^{2}=0.6766$ y la ecuación de la línea de tendencia es $\mathrm{Y}=0.4595 \mathrm{X}+13.24$. Cuando se correlacionan Tic y Tie de una selección de los días sin lluvia de este experimento, donde la diferencia entre ambos módulos es igual o superior a $3.8{ }^{\circ} \mathrm{C}$, el coeficiente de determinación es muy alto, $\mathrm{R}^{2}=0.9518$ y la ecuación de la línea de tendencia es $\mathrm{Y}=1.0354 \mathrm{X}$ 5.1205. En este caso la tendencia es como en los otros experimentos (pendiente positiva, Tie aumenta con Tic) pero la diferencia entre Tic y Tie permanece prácticamente estable en $4.0{ }^{\circ} \mathrm{C}$ independientemente de la temperatura del MC.

En el caso del experimento 7, como se puede ver en la figura 20, el conjunto de datos de correlación se ubican dentro de la zona cubierta por los datos del experimento 6. La tendencia en ambos casos es muy similar. La eficiencia de enfriamiento crece al aumentar Tic; aquí, el enfriamiento radiativo nocturno, que no estaba presente en el experimento 6 , produce un incremento en la diferencia media de temperatura de $0.2{ }^{\circ} \mathrm{C}$. Por ello, las líneas de tendencia en ambos casos corren de forma casi paralela, con la misma pendiente, pero con una diferencia aproximada de $0.2{ }^{\circ} \mathrm{C}$ inferior, en este caso, respecto al experimento 6. Corresponde, en este caso, un coeficiente de determinación alto $\mathrm{R}^{2}=0.7587$ y la ecuación de la línea de tendencia es $\mathrm{Y}=0.495 \mathrm{X}+11.91$.

La gráfica de correlación en la Figura 20 permite la comparación del comportamiento térmico de todos los sistemas evaluados experimentalmente bajo las mismas condiciones climáticas. De tal forma que, se puede estimar la temperatura del ME en cada uno de los experimentos para una temperatura dada de MC. Como ejemplo, se presentan en la Tabla 7 la temperatura media estimada en cada sistema cuando la temperatura media del $\mathrm{MC}$ es de $31^{\circ} \mathrm{C}$.

De la comparación de los experimentos 3 y 6 (este último, con datos seleccionados) se puede concluir que, el comportamiento térmico de los dos sistemas es muy similar, cuando se evalúa bajo condiciones climáticas semejantes. 
Figura 20 - Correlación entre las temperaturas medias del módulo de control (Tic) y las temperaturas medias del módulo experimental (Tie) en cada uno de los experimentos

Correlación entre Tic (MC) y Tie (ME)

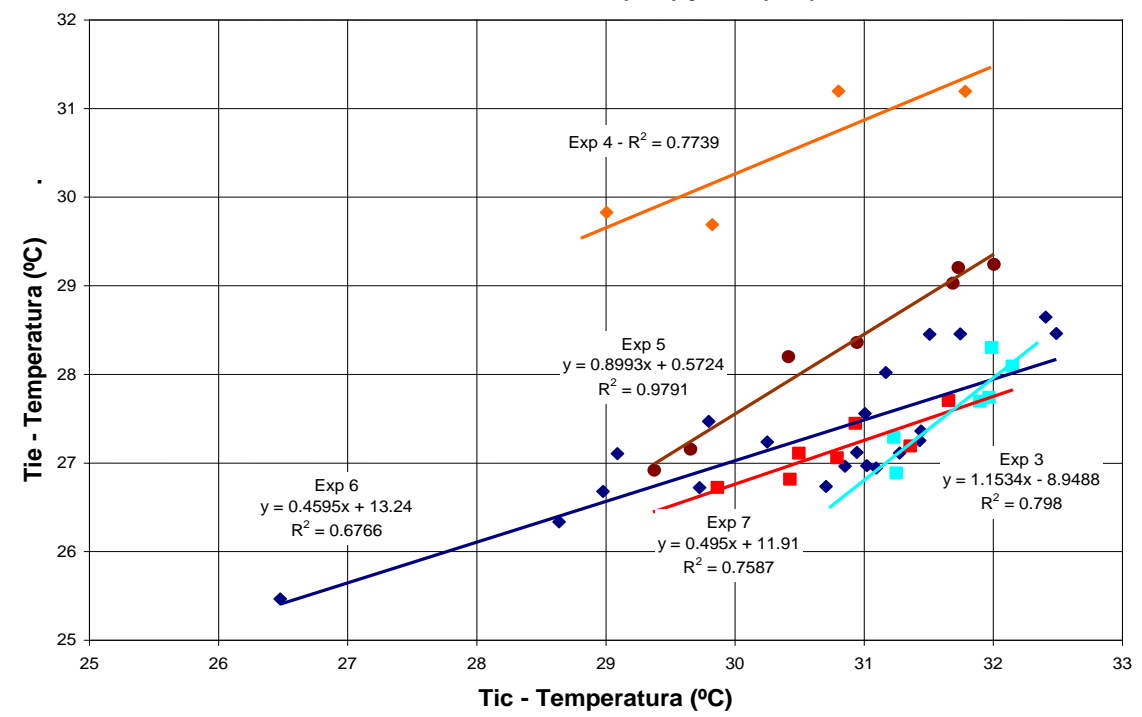

Tabla 7 - Valores de Tme cuando la temperatura media del MC es de $31{ }^{\circ} \mathrm{C}$

\begin{tabular}{c|lc}
\hline Exp & \multicolumn{1}{|c}{ Configuración de Módulo experimental } & Tme \\
\hline 3 & Masa térmica+enfriamiento evaporativo indirecto & $26.8^{\circ} \mathrm{C}$ \\
4 & Masa térmica+aislamiento & $30.8^{\circ} \mathrm{C}$ \\
5 & Masa térmica+enfriamiento radiativo & $28.5^{\circ} \mathrm{C}$ \\
6 & Masa térmica+enfriamiento evaporativo indirecto & $27.5^{\circ} \mathrm{C}$ \\
7 & Masa térmica+enfriamiento evaporativo indirecto + enfriamiento radiativo & $27.3^{\circ} \mathrm{C}$ \\
\hline
\end{tabular}

\section{Análisis del Potencial de Enfriamiento Medio (PEM)}

El potencial de enfriamiento medio (PEM) se define como "[...] la cantidad de energía por unidad de tiempo y de superficie, capaz de ser retirada por el sistema, obtenida como valor medio durante un periodo de 24 horas [...]" (GONZÁLEZ, 1997a, p. 214) (Ec. 1).

$\mathrm{PEM}=\mathrm{CPC} \mathrm{x}(\mathrm{Tm}$ control $-\mathrm{Tm}$ experimental $) \mathrm{x}$ $24 \mathrm{H} / \mathrm{A}$

Ec. 1

Donde:

CPC: Coeficiente de Pérdida de Calor del módulo (W/K);

Tm control: temperatura media del módulo de control en $24 \mathrm{~h}\left({ }^{\circ} \mathrm{C}\right)$;

Tm experimental: temperatura media del módulo experimental en $24 \mathrm{~h}\left({ }^{\circ} \mathrm{C}\right)$; y

A: área de la superficie efectiva del techo $\left(\mathrm{m}^{2}\right)$.

La metodología utilizada en este trabajo para determinar los valores de PEM para cada uno de los sistemas y días de monitoreo esta basada en los trabajos de Givoni (1981) y González (1989). Como ya ha sido explicado en el punto "descripción del montaje experimental", los módulos que se utilizan en la investigación tienen características constructivas y dimensiones idénticas a las utilizadas por González (1989) con lo cual se consideran iguales tanto el valor de "coeficiente de perdida de calor" $(\mathrm{CPC}=1.50 \mathrm{~W} / \mathrm{K})$ del módulo, como la superficie de techo en contacto con la superficie interior (área efectiva de enfriamiento $A=0.462 \mathrm{~m}^{2}$ ).

A continuación se presentan los resultados del cálculo de potencial de enfriamiento medio (PEM) de cada uno de los experimentos y el análisis de los mismos. En la Tabla 8 se presentan: los valores medios de PEM para cada experimento. En la Figura 21 podemos observar la distribución de los PEM resultado de cada uno de los días de cada experimento.

Una primera lectura de los valores medios de PEM resultado de cada experimento nos permite establecer una jerarquía de los sistemas estudiados. Así vemos que, el experimento 3 ha alcanzado el valor promedio más elevado (PEM=319.2 $\left.\mathrm{Wh} / \mathrm{m}^{2} \mathrm{dia}\right)$. Con el segundo valor más elevado de PEM se encuentra el experimento 7 (PEM=284.8 $\left.\mathrm{Wh} / \mathrm{m}^{2} \mathrm{dia}\right)$, un $10.8 \%$ inferior al alcanzado el experimento 3. El tercer valor más elevado de 
PEM recae sobre el experimento $6(\mathrm{PEM}=257.1$ $\left.\mathrm{Wh} / \mathrm{m}^{2} \mathrm{dia}\right)$, un $19.4 \%$ inferior al alcanzado el experimento 3. El experimento 5, con masa térmica y enfriamiento radiativo nocturno, se ubica en cuarto lugar con un valor promedio de $\mathrm{PEM}=198.1 \mathrm{Wh} / \mathrm{m}^{2}$ dia, un $37.9 \%$ inferior al alcanzado por el experimento 3 .

Una segunda lectura de estos resultados nos lleva a observar que, tanto en el experimento 6 como en el 7 , se alcanzan valores diarios de PEM superiores a los $300 \mathrm{Wh} / \mathrm{m}^{2}$ dia en algunos días, como sucede en el experimento 3. Sin embargo, la presencia de muchos días con precipitación, alta nubosidad y menores temperaturas, durante los experimentos 6 y 7, hacen que los sus promedios de PEM sean mucho menores que en el experimento 3. Como ya se ha discutido con anterioridad, la eficiencia de enfriamiento de los sistemas depende de las condiciones climáticas del lugar. Dado que los experimentos han sido monitoreados, no de forma simultanea, sino en periodos diferentes, entonces las condiciones climáticas también han sido diferentes. Esto trae como consecuencia que los valores medios obtenidos no sean directamente comparables entre si pues, la eficiencia de enfriamiento de ellos aumenta o disminuye con las condiciones climáticas del medio.

Tabla 8 - Estimación del potencial de enfriamiento medio

Estimacion Potencial de Enfriamiento Medio (PEM)

\begin{tabular}{c|c|c|c|c|c}
\hline \multicolumn{2}{c|}{$\mathrm{CPC}=1.5 \mathrm{~W} / \mathrm{K}$} & \multicolumn{4}{c}{ Superficie de techo $(\mathrm{A})=0.4624 \mathrm{~m}^{2}$} \\
\hline Experimento & Tic & Tie & DT & Wh/dia & Wh/m $\mathrm{m}^{2}$ ia \\
\hline 3 & 31.75 & 27.67 & 4.08 & 146.8 & 319.2 \\
\hline 4 & 30.35 & 30.48 & -0.12 & -4.4 & -9.6 \\
\hline 5 & 30.83 & 28.30 & 2.53 & 91.1 & 198.1 \\
\hline 6 & 30.57 & 27.29 & 3.28 & 118.3 & 257.1 \\
\hline 7 & 30.79 & 27.15 & 3.64 & 131.0 & 284.8 \\
\hline
\end{tabular}

Figura 21 - Potencial de enfriamiento medio (PEM) correspondiente a cada día de cada uno de los experimentos

POTENCIAL de ENFRIAMIENTO MEDIO (Wh/m2dia)

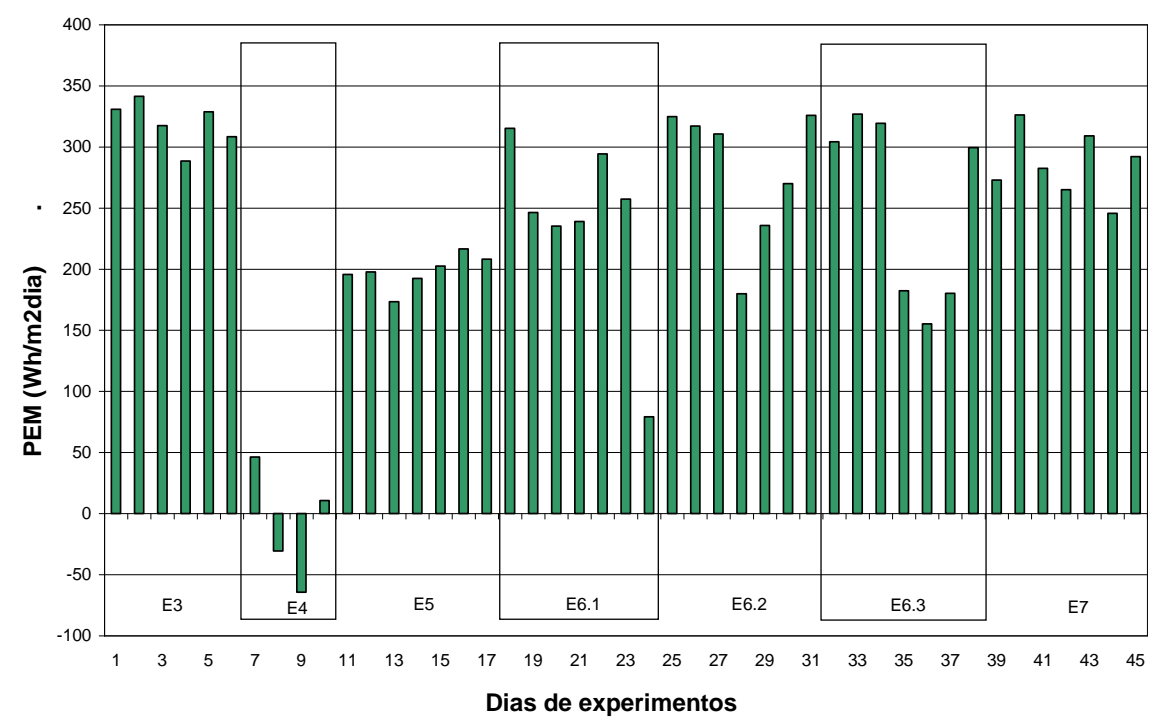

166 Gonzalez-Cruz, E. M.; Conzalez-Garcia, S. I. 
Un método de comparación más adecuado surge a partir del uso del gráfico de correlación entre las Tic-med y las Tie-med, en la Figura 20. Si se calculan los valores de PEM a partir de las ecuaciones de las rectas de regresión de los experimentos 3, 5, 6 y 7 para las temperaturas de $31{ }^{\circ} \mathrm{C}$ y $32{ }^{\circ} \mathrm{C}$ de Tic-med, (recordemos que el valor de Tic-med durante el experimento 3 fue de $31.75{ }^{\circ} \mathrm{C}$ ) encontramos dos posibles situaciones (Figura 22). En el primer caso el PEM del experimento 3 aumenta ligeramente y aun cuando los PEM de los experimentos 6 y 7 aumentan también, continúan siendo inferiores al experimento 3. En cambio, calculados a partir de $32{ }^{\circ} \mathrm{C}$, tanto el experimento 6 como el 7 resultan con valores de PEM superiores al experimento 3 . Cuando calculamos los PEM para la misma temperatura media de Tic que se registró durante el monitoreo del experimento 3 (Tic-med $=31.75{ }^{\circ} \mathrm{C}$ ), encontramos que el experimento 6 resulta con un $\mathrm{PEM}=306.9 \mathrm{Wh} / \mathrm{m}^{2} \mathrm{dia}$ ), tan solo $3.8 \%$ inferior al alcanzado en el experimento 3, y el experimento 7 tiene un $\mathrm{PEM}=322.7 \mathrm{Wh} / \mathrm{m}^{2} \mathrm{dia}$ ), es decir $1.1 \%$ superior al alcanzado en el experimento 3. En relación al experimento 5 , se observa que a medida que aumenta Tic-med también aumenta el PEM de este sistema, aun cuando de manera poco importante. No deja de ser interesante la eficiencia de enfriamiento del experimento 5 , que basa su potencial en la pérdida de calor que logra durante, tan solo 12 horas, cuando la masa térmica es expuesta al cielo nocturno.

Por otro lado, es importante recordar que la eficiencia de enfriamiento de los sistemas evaluados en los experimentos 3, 6 y 7, que basan su desempeño térmico en el aprovechamiento del enfriamiento evaporativo de manera indirecta, depende fundamentalmente de la depresión de la temperatura de bulbo húmedo (GIVONI, 1994).
Esto se comprueba al analizar los coeficientes de correlación entre la depresión de la temperatura de bulbo húmedo y el PEM para cada día de cada uno de los experimentos 3, 6 y 7 (Figura 23). Observamos coeficientes de determinación muy fuertes en los casos de los experimentos 3 y 6 $\left(\mathrm{R}^{2}=0.92\right.$ y $\mathrm{R}^{2}=0.95$ respectivamente), que dependen del enfriamiento evaporativo indirecto. Mientras que, se observa un coeficiente de determinación algo menor $\left(\mathrm{R}^{2}=0.8052\right)$ en el caso del experimento 7 , cuya eficiencia de enfriamiento no depende en su totalidad de la humedad del aire, sino también del estado del cielo. Estos resultados son totalmente coherentes con lo esperado. Este gráfico de correlación expresa que, la eficiencia de enfriamiento mayor se encuentra en el sistema evaluado en el experimento 7, mientras que, los sistemas evaluados en los experimentos 3 y 6 presentan eficiencias de enfriamiento muy similares entre si y ligeramente inferiores al experimento 7 .

\section{Conclusiones}

Se ha estudiado experimentalmente el comportamiento térmico de cuatro sistemas pasivos de enfriamiento, a partir de un nuevo dispositivo de techo-estanque. Entre las técnicas de enfriamiento pasivo aplicadas se encuentran el enfriamiento evaporativo indirecto (experimentos 3,6 y 7) y el enfriamiento radiativo nocturno (experimentos 5 y 7). En algunos casos, la utilización de pantallas de protección solar, aislamiento térmico y "agua confinada" como masa térmica, se han combinado con las técnicas de enfriamiento. También se ha evaluado el rol de la masa térmica sin enfriamiento pasivo (experimento 4).

Figura 22 - PEM ajustados por regresión lineal

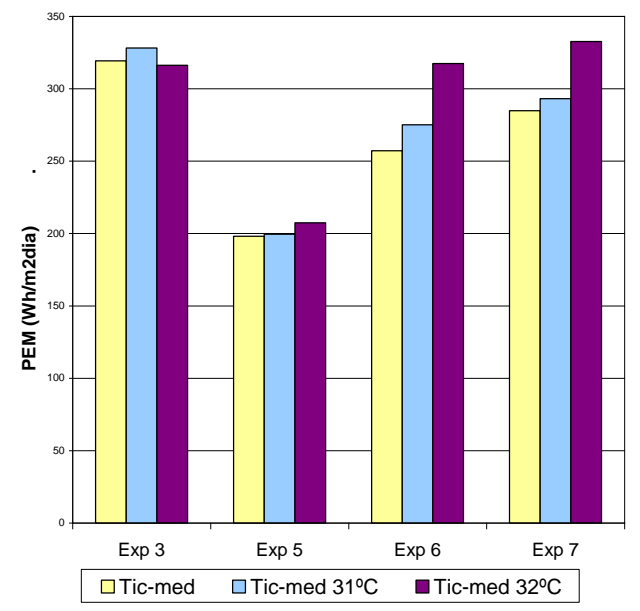


Figura 23 - Correlación entre la depresión de temperatura de bulbo húmedo (TBS-TBH) y el potencial de enfriamiento medio (PEM)

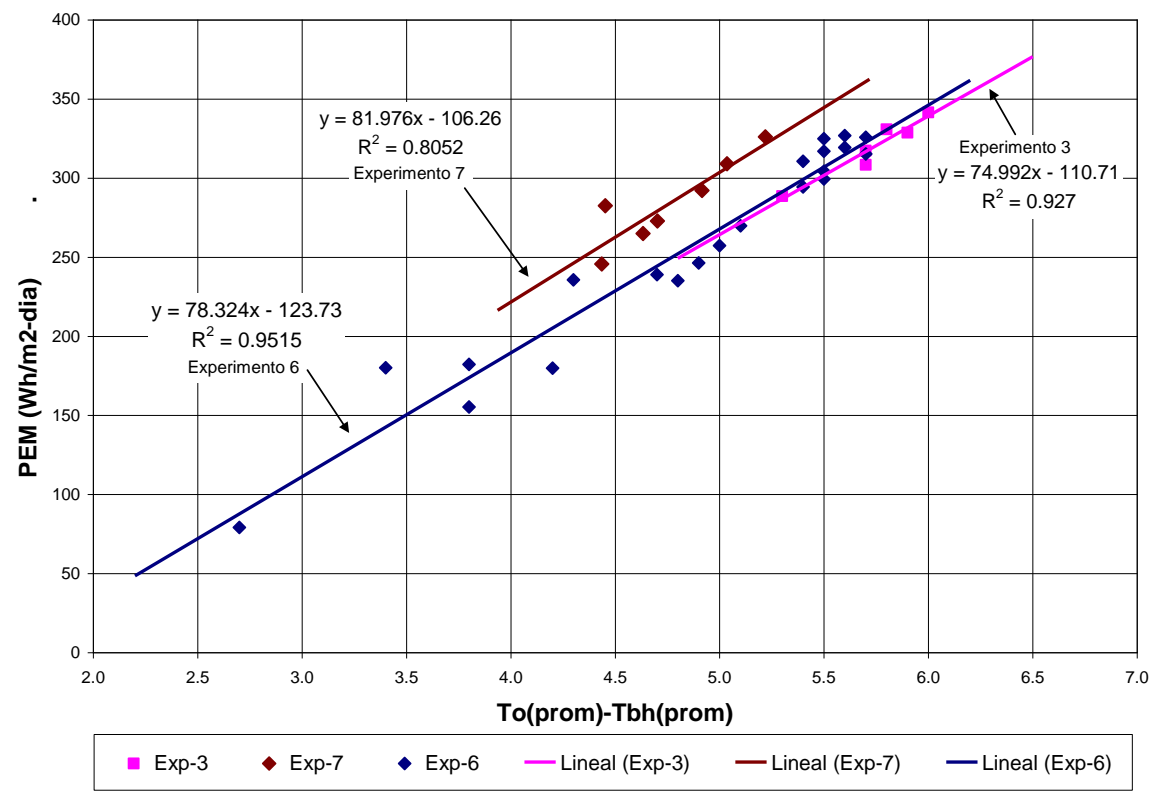

El análisis del comportamiento térmico de cada experimento ha permitido establecer la eficiencia de enfriamiento de las técnicas aplicadas. Se constata que en los experimentos 3, 5, 6 y 7, las temperaturas máximas del $\mathrm{ME}$ son siempre inferiores, no solo a la temperatura máxima del ambiente, sino también, a su temperatura media; las temperaturas medias del ME alcanzan valores semejantes o muy cercanos a las temperaturas mínimas exteriores; y las temperaturas mínimas del ME son siempre inferiores a las mínimas del ambiente.

La comparación de los resultados de los experimentos 3,6 y 7, a partir de valores obtenidos mediante las ecuaciones de regresión lineal, permite concluir que, la ventaja comparativa del experimento 3 sobre el 6 y el 7 (en eficiencia de enfriamiento) se limita a temperaturas inferiores a $32{ }^{\circ} \mathrm{C}$ de tmed en el MC. Sobre este valor de temperatura, tanto el caso 6 como el 7 resultan con mayor eficiencia de enfriamiento; esto se confirma al comparar los PEM de cada sistema, ajustados en función de sus ecuaciones de correlación.

Por otro lado, el efecto de la masa térmica se ha comprobado al observar los factores decrementales (FD) establecidos en relación con la amplitud de la temperatura interna del MC. Se ha registrado el menor FD (0.29) en el caso del experimento 4, donde la masa térmica constituida por 30 lts de agua confinada en el techo-estanque ha permanecido aislada todo el tiempo. Esa misma cantidad de masa térmica, en el experimento 3, con enfriamiento evaporativo indirecto, ha dado como resultado un valor promedio de FD de 0.39 semejante al observado en el experimento 5 (0.40). Mientras que, en los sistemas con agua confinada como masa térmica y enfriamiento evaporativo indirecto (experimento 6) o enfriamiento evaporativo indirecto más enfriamiento radiativo (experimento 7) se han observado valores de 0.34 y 0.36 respectivamente, inferiores a los del experimento 3 .

En relación con el potencial de enfriamiento medio de los sistemas evaluados, en la Tabla 9, se comparan los resultados obtenidos en este estudio con valores de PEM resultado de estudios semejantes realizados en Israel (GIVONI, 1981), y en Maracaibo, Venezuela (GONZÁLEZ, 1989; HINZ, 2006). Cuando se compara el experimento 3 de este estudio con los resultados de la serie 1 (HINZ, 2006), dos sistemas idénticos, vemos que el primero tiene un PEM de $319.2 \mathrm{Wh} / \mathrm{m}^{2}$ día y el segundo de $291.1 \mathrm{Wh} / \mathrm{m}^{2}$ día, evidentemente como consecuencia de haber sido realizados los estudios en condiciones climáticas diferentes. Sin embargo, la diferencia entre ellos puede verse dentro de los valores aceptables. Los valores de PEM de la serie dos (HINZ, 2006), donde se combina el efecto del enfriamiento evaporativo indirecto con la evapotranspiración de la vegetación y la sombra generada por ella, son los más elevados de los sistemas comparados. En segundo lugar se encuentran el experimento 3 y el caso de estanque de agua sobre techo de concreto expuesto en la noche y aislado en el día (GIVONI, 1981), este último evaluado en condiciones muy favorables del clima calido seco del desierto de Negev, en 
Israel. Conviene recordar, a la luz de estos resultados estadísticos, que tanto en el experimento 6 como el 7 se registraron valores máximos de PEM semejantes o superiores a los del promedio del experimento 3: $315.2 \mathrm{Wh} / \mathrm{m}^{2}$ día, 325.8 $\mathrm{Wh} / \mathrm{m}^{2}$ día, $326.9 \mathrm{Wh} / \mathrm{m}^{2}$ día y $326.1 \mathrm{Wh} / \mathrm{m}^{2}$ día, respectivamente. Sin embargo sus valores medios se ven reducidos de manera importante debido a valores muy bajos de PEM en días de lluvia.

De acuerdo con el análisis de correlación entre la depresión de temperatura de bulbo húmedo y el PEM, la mayor eficiencia de enfriamiento se observa en el sistema evaluado en el experimento 7, donde se combina el enfriamiento evaporativo indirecto, la masa térmica y el enfriamiento radiativo, mientras que, los sistemas evaluados en los experimentos 3 y 6 , solo con enfriamiento evaporativo indirecto, además de la masa térmica, presentan eficiencias de enfriamiento muy similares entre si y ligeramente inferiores al experimento 7 .

Puede concluirse que, la combinación de masa térmica, como la propuesta en este estudio, combinada con enfriamiento evaporativo indirecto, es una buena solución, con una elevada eficiencia de enfriamiento y que, además, su eficiencia aumenta a medida que las condiciones son más extremas. Una eficiencia aún mayor se consigue al implicar el enfriamiento radiativo pero evidentemente a un mayor costo y complejidad del sistema. Estos sistemas pueden resultar aún más convenientes en climas calientes sub-húmedos y en especial en climas calientes y secos donde la diferencia promedio entre la temperatura de bulbo seco y bulbo húmedo es mayor que la observada durante los experimentos.

De estos resultados se puede deducir que la aplicabilidad de un sistema como el evaluado en el experimento 3 conviene más para unas condiciones climáticas como las de Maracaibo, en cambio, los sistemas de los experimentos 6 y 7 resultan más favorables en condiciones climáticas más calientes y secas. No debe perderse de vista que este nuevo dispositivo de techo-estanque con masa térmica confinada puede ser utilizado para el enfriamiento pasivo, como se ha demostrado, pero también, como sistema pasivo de calentamiento o simplemente como masa térmica en climas o periodos del año donde no es requerido ni enfriamiento ni calentamiento.

Tabla 9 - Valores de PEM de sistemas estudiados, comparados otras experiencias en Venezuela e Israel

\begin{tabular}{l|l|c}
\hline \multicolumn{1}{c|}{ Experiencias } & \multicolumn{1}{|c}{ Descrpción del sistema de enfriamiento } & $\begin{array}{c}\text { PEM } \\
\text { (Wh/m } \mathbf{m}^{2} \text { dia) }\end{array}$ \\
\hline Gonzalez S. (2010) Exp-3 & Techo-estanque metálico con agua, ventilado y sombreado (EEI+PS) & 319.2 \\
\hline Gonzalez S. (2010) Exp-5 & $\begin{array}{l}\text { Techo-estanque metálico con agua confinada expuesto en la noche y } \\
\text { aislamiento diurno (MT+ER+AT) }\end{array}$ & 198.1 \\
\hline Gonzalez S. (2010) Exp-6 & $\begin{array}{l}\text { Techo-estanque metálico con agua confinada y agua ventilada y } \\
\text { sombreada (EEI+MT+PS) }\end{array}$ & 257.1 \\
\hline Gonzalez S. (2010) Exp-7 & $\begin{array}{l}\text { Techo-estanque metálico con agua confinada y agua ventilada y } \\
\text { sombreada, Expuesto en al noche (EEl+ER+MT+PS) }\end{array}$ & 284.8 \\
\hline Hinz E. (2006) Serie 1 & $\begin{array}{l}\text { Estanque con agua en techo protegido del sol y ventilación natural. } \\
\text { (EEI+PS) }\end{array}$ & 291.1 \\
\hline Hinz E. (2006) Serie 2 & $\begin{array}{l}\text { Estanque con agua en techo protegido del sol con vegetación sobre } \\
\text { poliestireno y ventilación natural. (EEI+CV+PS) }\end{array}$ & 366.3 \\
\hline González E. (1989) & $\begin{array}{l}\text { Estanque metálico, con agua confinada en techo, expuesto al cielo } \\
\text { durante la noche, aislado durante el día. (MT+ER+AT)) }\end{array}$ & 275.5 \\
\hline González E. (1989) & $\begin{array}{l}\text { Techo de concreto con tierra y capa de vegetación, riego dos veces al } \\
\text { dia. (MT+CV+EEI) }\end{array}$ & 186.0 \\
\hline Givoni B. (1981) & $\begin{array}{l}\text { Estanque de agua sobre techo de concreto expuesto en la noche y } \\
\text { aislamiento diurno. (MT+EEl+ER+AT) }\end{array}$ & 320.0 \\
\hline Givoni B. (1981) & $\begin{array}{l}\text { Estanque de agua sobre techo de concreto, ventilado y sombreado. } \\
\text { (MT+EEI+PS) }\end{array}$ & 274.0 \\
\hline
\end{tabular}

Nota: Leyenda:

EEI: enfriamiento evaporativo indirecto;

PS: protección solar;

MT: masa térmica;

ER: enfriamiento radiativo;

AT: aislamiento térmico; y

CV: capa vegetal. 


\section{Referencias}

BALARAS, C. A. The Role of Thermal Mass on the Cooling Load of Buildings: an overview of computational methods. Energy and Building, v. 24, n. 1, p. 1-10, 1996.

BRAVO, G.; GONZÁLEZ, E. Thermal Comfort in Naturally Ventilated Spaces and Under Indirect Evaporative Passive Cooling Conditions in Hot: humid climate. Energy and Building, v. 63, p. 79-86, ago. 2013.

CÁMARA VENEZOLANA DE LA INDÚSTRIA ELÉCTRICA. Estadísticas Consolidadas. 2007.

Disponible en: <www.caveinel.com>. Acceso: 04 nov. 2013.

DORNELLES, K. A.; RORIZ, M. Thermal Inertia, Confort and Energy consumption in Buildings: a case study in Sao Paulo State-Brasil.

International Journal for Housing Science and Its Applications, v. 28, n. 2, p. 153-162, 2004.

GIVONI, B. Experimental Studies on Radiant and Evaporative Cooling of Roofs. In:

PROCEEDINGS OF THE INTERNATIONAL PASSIVE AND HYBRID COOLING

CONFERENCE, Miami Beach, 1981.

Proceedings... Miami Beach, 1981. p. 279-283, 1981.

GIVONI, B. Indoor Temperature Reduction by Passive Cooling Systems. Solar Energy, v. 85, n. 8, p. 1692-1726, aug. 2011.

GIVONI, B. Passive and Low Energy Cooling of Buildings. New York: Van Nostrand Reinhold, 1994.

GIVONI, B.; GONZÁLEZ, E. Thermal Performance of Indirect Evaporative Cooling in a Tropical Climate. In: ASES CONFERENCE, Buffalo, NY, 2009. Proceedings... Buffalo, NY, 2009.

GONZÁLEZ, E. et al. Desempeño Térmico de la VBP-1: temperaturas características, factor decremental y retraso térmico. In: SEMANA NACIONAL DE ENERGÍA SOLAR, Veracruz, Mexico, 2006. Memorias... Veracruz, Mexico. 2006.

GONZÁLEZ, E. Enfriamiento Evaporativo Indirecto en Clima Tropical Húmedo: dos casos de estudio. In: CONGRESO LATINOAMERICANO SOBRE CONFORT Y COMPORTAMIENTO TÉRMICO DE EDIFICACIONES, 4., Ciudad de Mexico, 2005, Memorias... COTEDI-2005, Ciudad de Mexico, Mexico. 2005.
GONZÁLEZ, E. Étude de Matériaux et de Techniques du Bâtiment Pour la Conception Architecturale Bioclimatique en Climat Chaud et Humide. Thèse (Doctorat en Energétique) l'École des Mines des Paris, Paris, 1997a.

GONZÁLEZ, E. Evaluación de Sistemas Pasivos de Enfriamiento y Su Aplicación en el Diseño de Viviendas. 1989. Informe de investigación CONDES, Universidad de Zulia, Maracaibo, Venezuela.

GONZÁLEZ, E. Técnicas de Enfriamiento Pasivo. Resultados Experimentales en el Clima Cálido y Húmedo de Maracaibo, Venezuela. Información Tecnológica, La Serena, Chile, v. 8, n. 5, p. 99103. $1997 \mathrm{~b}$

GONZÁLEZ, E. et al. VBP-1: a sustainable urban house for low-income family in a tropical climate. In: PASSIVE AND LOW ENERGY ARCHITECTURE CONFERENCE 2000, Cambridge, UK, 2000. Proceedings... Cambridge, UK, 2000.

HINZ, E. Estudio del Comportamiento Térmico de Un Sistema Pasivo de Enfriamiento Evaporativo Indirecto Con Cobertura Vegetal en Un Clima Tropical. Trabajo de Suficiencia Investigadora, Diploma de Estudios Avanzados (DEA), Doctorado en Ciencias Ambientales, Universidad Politécnica de Madrid, Madrid, 2006.

KHARRUFA, S. N.; ADIL, Y. Roof Pond Cooling of Buildings in Hot Arid Climates. Building and Environment, v. 43, n. 1, p. 82-89, 2008.

KRÜGER, E.; GONZÁLEZ, E.; GIVONI, B. Effectiveness of Indirect Evaporative Cooling and Thermal Mass in a Hot Arid Climate. Building and Environment, v. 45, p. 1422-1433, 2010.

LIGGETT, R.; MILNE, M. Climate Consultant V-5.2. UCLA Energy Design Tools Group. 2011. Disponible en: <http://www.energy-designtools.aud.ucla.edu/>. Acceso en: 14 nov. 2013.

MARSH, A. Weather Tool V-200. SQUARE ONE research, Centre for Research in the Built Environment, Cardiff, Wales CF10 3NB, 2005.

RINCÓN, J.; ALMAO, N.; GONZÁLEZ, E. Experimental and Numerical Evaluation of a Solar Passive Cooling System Under Hot and Humid Climatic Conditions. Solar Energy, v. 71, n. 1, p. 71-80, 2001.

SÁNCHEZ, L. Evaluación de Un TechoEstanque Como Sistema de Enfriamiento Pasivo en Un Clima Cálido Sub-húmedo. Tesis (Maestría en Diseño Bioclimática) - Universidad de Colima, México, 1993.

170 Gonzalez-Cruz, E. M.; Conzalez-Garcia, S. I. 
SOEVARTO, V. I. A New Approach to Passive Design For Residential Buildings in a Tropical Climate. In: PASSIVE AND LOW ENERGY ARCHITECTURE CONFERENCE 1999, Brisbane, Australia, 1999. Proceedings..., Brisbane, Australia, 1999. p. 369-374.

SZOKOLAY, S. V. Dilemas of Warm-Humid Climate House Design: heavy vs. lightweight + cooling effect of air movement. In: PASSIVE AND LOW ENERGY ARCHITECTURE CONFERENCE 2000, Cambridge, UK, 2000. Proceedings... Cambridge, UK, 2000. p. 144-149..

TANG, R.; ETZION, Y. On Thermal Performance of an Improved Roof Pond For Cooling Buildings. Building and Environment, v. 39, n. 2, p. 201209, 2004.

TANG, R.; ETZION, Y. Cooling Performance of Roof Ponds With Gunny Bags Floating on Water Surface as Compared With a Movable Insulation. Renewable Energy, v. 30, n. 9, p. 1373-1385, 2005.
TANG, R.; ETZION, Y.; ERELL, E. Experimental Studies on a Novel Roof Pond Configuration For the Cooling of Buildings. Renewable Energy, v. 28, n. 10, p. 1513-1522, 2003.

\section{Agradecimiento}

Se agradece a CAPES por la beca otorgada al Prof. Eduardo Manuel González Cruz dentro del "Programa CAPES/Professor visitante do exterior - PVE", lo que hace posible presentar este trabajo en ENCAC 2013. Este trabajo se basa en la Tesis de Maestría de la Arq. Sabrina González, dentro del Programa de Estudios: "VIII Master en Energías Renovables: Arquitectura y Urbanismo. La ciudad Sostenible" de la Universidad Internacional de Andalucía, sede Iberoamericana Santa María de La Rábida, España.

\section{Eduardo Manuel González-Cruz}

Facultad de Arquitectura y Diseño, Instituto de Investigaciones | Universidad del Zulia | Av. 16 (Guajira) con calle 67 (Cecilio Acosta) | Maracaibo, Venezuela | Tel.: +58 (414) 6103089 | E-mail: edugoncruz@gmail.com

Sabrina Isabella González-Garcia

VIII Máster en Energías Renovables: Arquitectura y Urbanismo, La ciudad Sostenible | Universidad Internacional de Andalucia | Sede Iberoamericana Santa María de la Rábida | 21819 | Paraje La Rábida | Palos de La Frontera - Huelva - España | Tel.: +58 (414) 6155087 | E-mail: sabrinaigonzalez@gmail.com

\section{Revista Ambiente Construído}

Associação Nacional de Tecnologia do Ambiente Construído

Av. Osvaldo Aranha, $99-3^{\circ}$ andar, Centro

Porto Alegre - RS - Brasil

CEP $90035-190$

Telefone: +55 (51) 3308-4084

Fax: +55 (51) 3308-4054

www.seer.ufrgs.br/ambienteconstruido

E-mail: ambienteconstruido@ufrgs.br 